\title{
Novel biomaterial strategies for controlled growth factor delivery for biomedical applications
}

\author{
Zhenming Wang ${ }^{1}$, Zhefeng Wang ${ }^{1}$, William Weijia Lu², Wanxin Zhen ${ }^{1}$, Dazhi Yang ${ }^{1}$ and Songlin Peng ${ }^{1}$ \\ Growth factors (GFs) are soluble proteins secreted by cells that have the ability to regulate a variety of cellular processes and \\ tissue regeneration. However, their translation into clinical applications is limited due to their short effective half-life, low \\ stability, and rapid inactivation by enzymes under physiological conditions. To maximize the effectiveness of GFs and their \\ biologically relevant applicability, a wide variety of sophisticated bio-inspired systems have been developed that augment tissue \\ repair and cellular regeneration by controlling how much, when, and where GFs are released. Recently, protein immobilization \\ techniques combined with nanomaterial carriers have shown promise in mimicking the natural healing cascade during tissue \\ regeneration by augmenting the delivery and effectiveness of GFs. This review evaluates the latest techniques in direct \\ immobilization and relevant biomaterials used for GF loading and release, including synthetic polymers, albumin, \\ polysaccharides, lipids, mesoporous silica-based nanoparticles (NPs), and polymeric capsules. Specifically, we focus on \\ GF-encapsulated NPs in functionalized microporous scaffolds as a promising alternative with the ability to mimic extracellular \\ matrix (ECM) hierarchical architectures and components with high cell affinity and bioactivity. Finally, we discuss how these \\ next-generation, advanced delivery systems have been used to enhance tissue repair and regeneration and consider future \\ implications for their use in the field of regenerative medicine.
}

NPG Asia Materials (2017) 9, e435; doi:10.1038/am.2017.171; published online 6 October 2017

\section{INTRODUCTION}

Growth factors (GFs) as powerful therapeutic agents for tissue regeneration

GFs are soluble proteins secreted by cells that have the ability to regulate a wide range of cellular behaviors, such as proliferation, migration and differentiation, by binding to specific transmembrane receptors on target cells. ${ }^{1}$ The chemical identity, duration, concentration and sequence of multiple GFs and the particular extracellular matrix (ECM) in which they are applied can have a significant effect on cell fate and the responsiveness of wound healing. ${ }^{2,3}$ In particular, the context in which GFs are used, for example, alone or in various combinations, has shown great therapeutic potential in preclinical models and clinical applications. Some of the more widely studied GFs in tissue regeneration and repair are those involved in angiogenesis and osteogenesis. These include vascular endothelial growth factors (VEGFs), fibroblast growth factor (FGF) and placental growth factors (PIGFs), which are involved in angiogenesis and play a crucial role in the repair of most tissues; ${ }^{4-6}$ insulin-like growth factors (IGFs), transforming growth factor beta (TGF- $\beta$ ), bone morphogenetic proteins (BMPs), particularly BMP-2, BMP-4 and BMP-7, which are the most extensively used osteogenic proteins for inducing new bone formation in bone defects and ectopic sites; ${ }^{7-10}$ and platelet-derived growth factor-BB (PDGF-BB), which plays a pivotal role in the formation of periodontal tissue and the recruitment of osteoblast progenitors and stem cells. ${ }^{6,11}$ To date, the only GFs that have been clinically approved by the Food and Drug Administration (FDA) are BMP-2 and BMP-7 for lumbar spine fusion and bone fracture and PDGF-BB for the enhancement of granulation tissue formation.

Clinical limitations of GFs

As summarized above, a number of GFs have shown great therapeutic potential in clinical trials, and some have been approved for specific biomedical applications. However, only a relatively small subset has achieved commercial success due to safety and cost-effectiveness issues. A major limitation of natural GFs is their short effective halflife, low protein stability, and rapid deactivation of their specialized properties by enzymes at body temperature. As an example, FGF-1 presented intrinsically low stability, with an active half-life of only $1 \mathrm{~h}$ in serum at $37^{\circ} \mathrm{C},{ }^{12}$ whereas the half-life of basic fibroblast growth factor (bFGF) is $3 \mathrm{~min}^{13}$ and that of VEGF is only $50 \mathrm{~min}^{14}$ following intravenous injection. Moreover, direct injection of GFs in high enough doses in order to reach and sustain a sufficiently high local concentration to be effective may result in side effects in vivo. Clinical doses of BMP-2 in lumbar spinal arthrodesis is associated with an increased risk of new cancer development. ${ }^{15}$ Similarly, VEGF has been shown to trigger edema and systemic hypotension in cardiovascular

${ }^{1}$ Department of Spine Surgery and Institute of Orthopedic Research, Shenzhen People's Hospital, Jinan University Second College of Medicine, Shenzhen, China and ${ }^{2}$ Department of Orthopaedics \& Traumatology, The University of Hong Kong, Hong Kong, China

Correspondence: Dr D Yang or Dr S Peng, Department of Spine Surgery and Institute of Orthopedic Research, Shenzhen People's Hospital, Jinan University Second College of Medicine, Shenzhen 518001, China.

E-mail: yangdazhi@medmail.com.cn or songlin824@gmail.com

Received 15 March 2017; revised 13 June 2017; accepted 10 July 2017 
a

Physical encapsulation/immobilization b

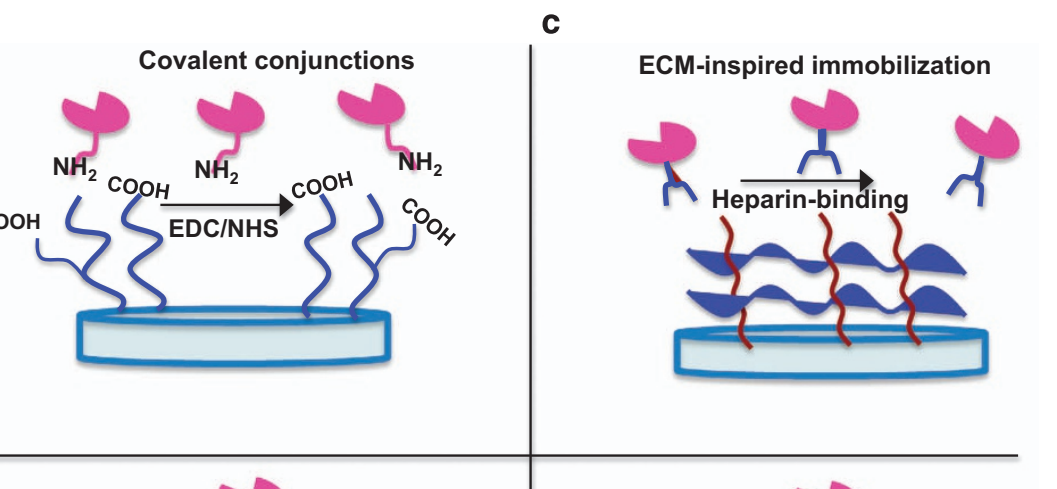

2

1

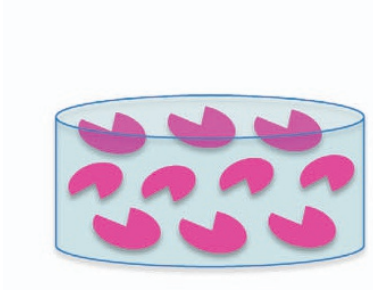

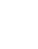

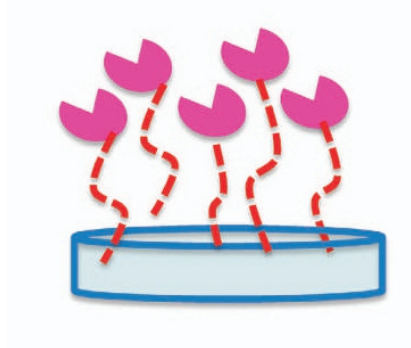

3
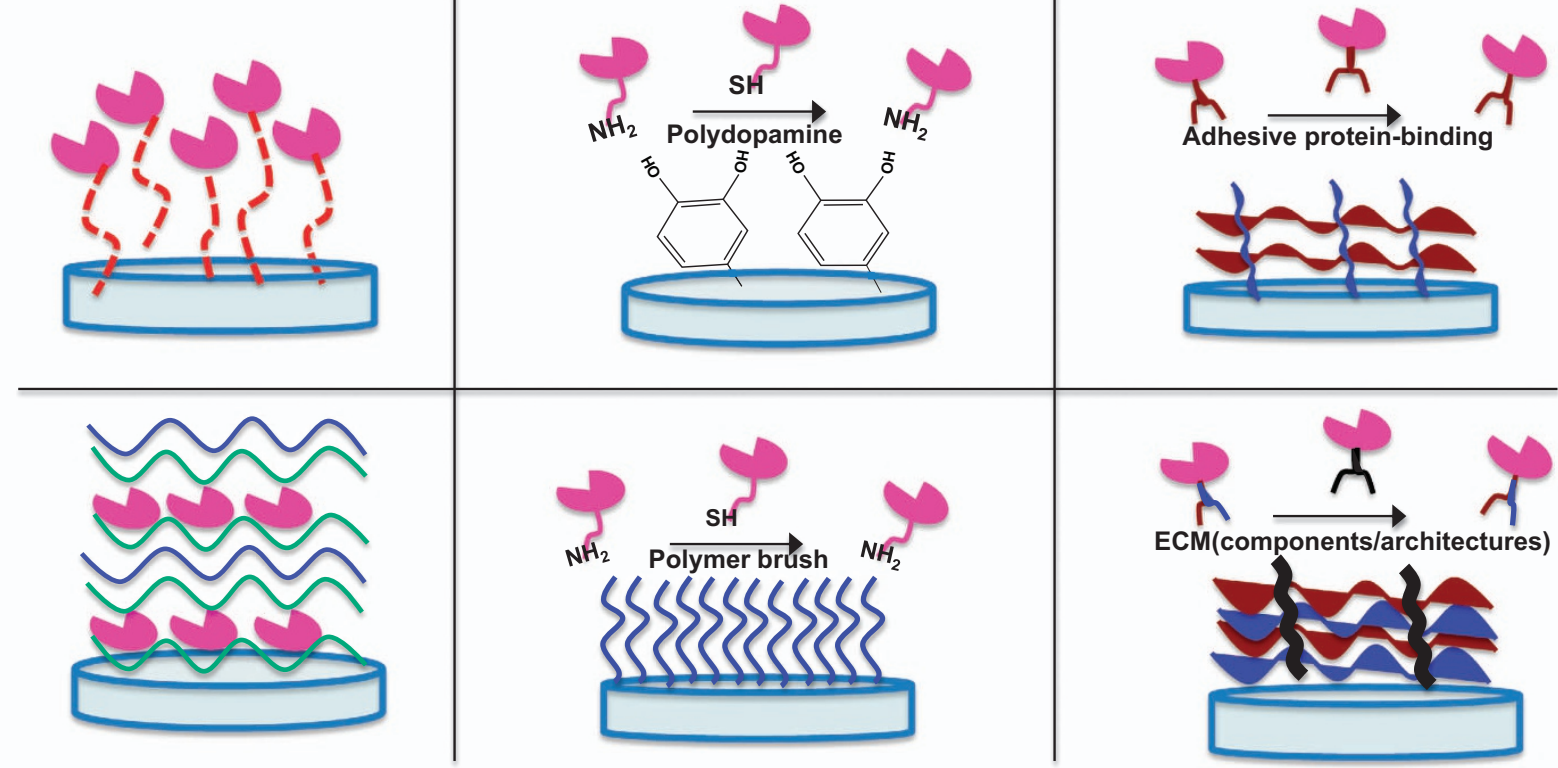

ECM(components/architectures)

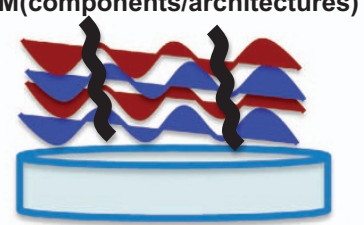

Figure 1 The direct approaches for the immobilization/encapsulation of growth factors (GFs) to biomaterials. (a) Physical immobilization techniques. (b) Nonselective covalent immobilization of GFs through their functional residues. (c) Extracellular matrix (ECM)-inspired immobilization reactions used for the orientation of GFs on the surfaces of biomaterials.

and peripheral applications, raising serious concerns for its use in bone engineering. ${ }^{16}$ In addition to serious side effects, the lack of costeffective methods for producing recombinant GFs at vastly supraphysiological levels is another major obstacle that may limit their biomedical applications.

\section{Advanced drug delivery systems in tissue engineering}

Collectively, the safety and cost-effectiveness issues of GFs mainly derive from their initial burst release kinetics and rapid clearance at injured sites when they are administered without appropriate spatiotemporal control. The short half-lives of the GFs, their relatively large size and their potential toxicity at high systemic doses suggest that conventional delivery techniques are not well suited to the clinical administration of GFs. To address these limitations, more sophisticated delivery systems that allow for controlled, precise, sustained, and localized release of these proteins have been developed, enabling optimal doses and spatial/temporal gradients in localized sites for effective tissue regeneration. ${ }^{17}$ Recently, nano-delivery systems have been developed that are capable of providing an artificial ECM for cell attachment and penetration while maintaining a 3D network on which tissue regeneration can be facilitated. ${ }^{18,19}$ In this review, we will focus on the most important aspects of these delivery mechanisms. First, we will discuss how GFs can be grafted onto appropriate biomaterials using advanced techniques, namely, directive immobilization. Second, we will highlight examples of biodegradable NPs, particularly synthetic polymers, albumin, polysaccharides, lipids, mesoporous silica-based nanocarriers, polymeric capsules and other nanocarriers for GF loading and release. Third, we will present examples of functionalized GF-encapsulated NP scaffolds and discuss their use in tissue regeneration enhancement in recent years. Finally, we will provide perspectives on rational design, which pools resources to accelerate the production of clinically available devices for regenerative engineering.

\section{IMMOBILIZATION AND RELEASE OF GFS IN DIRECT APPROACHES FOR BIOMEDICAL APPLICATIONS}

For direct GF delivery, three distinct strategies have been pursued in the past few years for biomaterial presentation of GFs in tissue engineering: physical, covalent, and bioaffinity GF immobilization. ${ }^{20}$ There are three types of direct binding of GFs in the carrier matrix, as summarized in (Figure 1). Table 1 presents a detailed description of some of the most relevant studies on direct factor delivery systems for biomedical applications.

\section{Physical encapsulation/immobilization of GFs}

Physical encapsulation of GFs in the delivery system. The simplest method to encapsulate GFs into a 3D polymer matrix is to mix the factors within the polymers before their gelation or solidification 


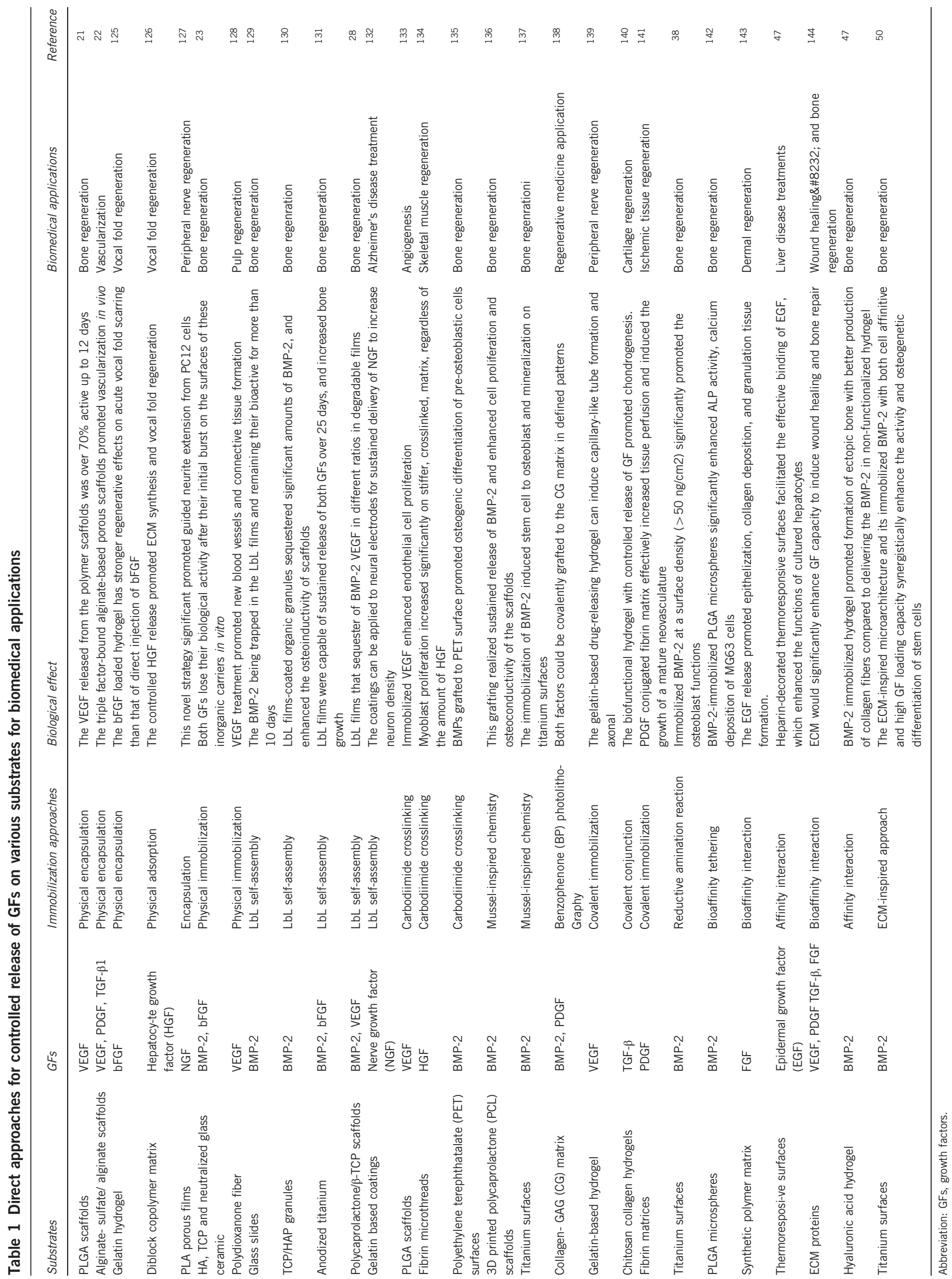


(Figure 1a1). This approach has the advantages that the optimized properties of scaffolds are not substantially affected by the presence of factors, and the bioactivity of GFs can be retained during their incorporation. For example, Murphy et al. ${ }^{21}$ have developed VEGFencapsulated poly(lactic-co-glycolic acid) (PLGA) scaffolds by introducing the GF into a gas foaming/particulate leaching process and showing that the VEGF released from the polymer scaffolds was over $70 \%$ active for up to 12 days. Freeman et al. ${ }^{22}$ have synthesized alginate-sulfate/alginate scaffolds that incorporated VEGF, PDGF-BB and TGF- $\beta 1$, which were bound to alginate-sulfate with an affinity similar to heparin, thus enabling the sequential delivery of three distinct GFs. The results demonstrated that vascularization within the triple factor-bound, alginate-based porous scaffolds was superior to that found in scaffolds consisting only of a single growth factor following subcutaneous implantation in rats. To our knowledge, such direct GF encapsulation can be achieved by impregnation, diffusion of the pre-formed matrix in very small volumes of GF solutions or immersion of the matrix in a GF solution. However, this incorporation approach is inefficient since only a small fraction of GFs can be bound in this system; in addition, the GFs have unpredictable release profiles.

Absorption of GFs on the surfaces of the matrix. Over the last two decades, the physical immobilization of GFs onto various matrices has attracted much attention because it is easy to accomplish under mild conditions at room temperature; however, this approach can frequently result in the inefficient retention of stable soluble protein and poorly controlled delivery (Figure 1a2). When Ziegler et al. ${ }^{23}$ directly immobilized BMP-2 and bFGF onto synthetic bone implants (HA, TCP and neutralized glass ceramic) utilizing the physical approach, they found that both GFs lost their biological activity after their initial burst on the surfaces of these inorganic carriers in vitro. The BMP-2 released from the inorganic carriers during the initial period led to a significant increase in the activity of ALP and the proliferation of human primary osteogenic sarcoma cells and these effects were attributed to the unbound bioactive protein. However, cells cultured in protein released from carriers after $24 \mathrm{~h}$ of adsorption did not increase the expression of ALP or other osteogenic activity. These studies underscore the need for more advanced methods that strengthen the immobilization of GFs to various implant surfaces or their encapsulation within carriers for long-term retention of osteoinductive activity.

Reyes et al. ${ }^{24}$ developed brushite cement and brushite/PLGA composite systems that controlled the release rate of PDGF, TGF- $\beta 1$ and VEGF to enhance bone regeneration. PDGF and TGF- $\beta 1$ were directly adsorbed onto pure brushite cement implants, whereas VEGF pre-loaded PLGA microspheres were incorporated in brushite scaffolds. In vivo results indicated that PDGF and TGF- $\beta 1$ were delivered more rapidly from brushite cement implanted in intramedullary defects in rabbit femurs than VEGF. Approximately $40 \%$ of the PDGF and TGF- $\beta 1$ was released in the first $24 \mathrm{~h}$ with release rates of both GFs dropping to $\sim 5.5 \%$ per day during the next 6 days. A total release of $90 \%$ of the GF was achieved after three weeks. In contrast, VEGF pre-encapsulated in PLGA microspheres and incorporated in brushite implants showed a more controlled release profile. A steady release of $7 \%$ per day was detected within the first week, and the release dropped by $1.2 \%$ per day during the following three weeks. A total release of almost $80 \%$ of the VEGF was accomplished within two months. Notably, the three types of observed GF release profiles showed a high correlation between in vivo and in vitro conditions. Therefore, the incorporation of GF-loaded polymeric microspheres in scaffolds could enable a sustained and more stable release of GFs from scaffolds in vivo compared to other configurations.

Layer by layer self-assembly. Although the direct adsorption of GFs on substrates was the first approach for the delivery of multiple GFs, it proved to be difficult in regulating the release rates of multiple GFs in a programmable manner (Figure 1a3). Therefore, alternative strategies can be employed to address this situation by incorporating multiple strata from the top to the bottom of the structure, such as bilayered or tetralayered coatings, for the spatial and temporal control of the delivery of multiple drugs within the healing tissue. The method, known as the Layer-by-Layer (LbL) approach, frequently relies on electrostatic interactions between oppositely charged polyelectrolytes and GFs to deposit functional polymer coatings on surfaces of varying composition and shape. ${ }^{25,26}$ Particularly relevant to protein delivery, the LbL approach takes advantage of facile aqueous baths for the construction of functional coatings; these baths have the potential to preserve fragile, soluble protein activity compared to typical methods that utilize harsh organic solvents for the fabrication of protein delivery systems.

The use of multi-layer biotechnology allows the design of GF vehicles with optimal release rates to the mimic GF profiles normally present during various types of tissue regeneration. For example, Hammond et al. ${ }^{27}$ first prepared tetralayer architectures by the LbL technique to capture and deliver GFs on the microgram-scale for a multi-week period, which resulted in defectively stimulating host cell behaviors in vivo. The LbL architecture was created on a 3D scaffold (on glass surface) that was repeatedly dipped (100 times) with tetralayer units consisting of (1) poly( $\beta$-aminoester) (positively charged polymer), (2) chondroitin sulfate (negatively charged polysaccharide), (3) BMP-2 (positively charged protein) and (4) chondroitin sulfate. In vitro results showed that $80 \%$ of the BMP-2 was released over 2 days (with minimal burst release) and that a sustained release of the remaining $20 \%$ of encapsulated GFs occurred over a period of approximately 2 weeks. In a follow up study, LbL films were constructed with tunable BMP-2 and VEGF delivery in degradable $\{$ poly( $\beta$-amino ester)/polyanion/growth factor/polyanion\} tetralayer repeat films, where the polyanion used for BMP-2 and VEGF adsorption are poly(acrylic acid) and chondroitin sulfate. ${ }^{28}$ No burst release of either GF was found in this system; the release of BMP-2 was sustained over a period of 2 weeks, whereas VEGF was discharged from the layers only during the first 8 days. In vivo results indicated that the mineral density of ectopic bone induced by BMP-2/VEGF LbL films was $\sim 33 \%$ higher than that of single GF entrapped layers. Another study recently utilized laponite clay as interlayer barriers in LbL films, which acted as a dual-purpose biomimetic microenvironment that sequentially released an antibiotic, followed by the release of BMP-2 for orthopedic implant applications. ${ }^{29}$ These studies suggested that the LbL approach could serve as a promising delivery system for the precisely controlled release of multiple drugs by employing components with different hydrolytic degradation rates.

\section{Covalent conjunctions}

Covalent immobilization (Figure 1b) of GFs to biomaterials can eliminate the possibility of initial burst release and has emerged as a promising approach for improving the stability and persistence of GFs when delivered into cells or tissues. ${ }^{30}$ Covalent immobilization is warranted when the binding biomolecules are incapable of adsorption on the substrate surfaces or when the GF adsorbs by weak physisorption forces due to improper orientation and conformation. By contrast, covalently immobilized GFs most often involve chemical/ 
enzymatic reactions between proteins and functionalized surfaces, offering important control of the amount, orientation, retention, and distribution of GFs in solid matrices, achieving GF delivery that is both localized and sustained. When the GF is chemically bound to the biomaterials, its desorption rate is controlled by enzymatic or hydrolytic cleavage of the chemical bond that grafts it to the matrix, facilitating the establishment of multiple release models for GFs, such as linear, pulsatile, or sequential protein release profiles. In addition, covalent conjunction can prolong GF availability and reduce the amount of protein required, thereby potentially increasing the therapeutic efficiency of biomaterials for enhanced tissues regeneration at reduced costs.

Carbodiimide coupling immobilization. Carbodiimide coupling chemistry presents one of the most popular approaches for covalently grafting GFs to various substrates due to its simplicity, high conjugation ratio, low cost and mild reaction conditions (Figure 1b1). ${ }^{31}$ Among these coupling reagents, the prevalent crosslinking molecule is similar to 1-ethyl-3-(3-dimethylamminopropyl) carbodiimide (EDC) for bioconjugation purposes. EDC mediates the conjugation reaction between carboxylic acid groups and amino groups, producing the formation of stable intermolecular amide bonds in aqueous environments at physiological $\mathrm{pH} .{ }^{32}$ Carbodiimide coupling reactions may engage the amino groups in the lysine residues and $\mathrm{N}$-terminus of GFs. However, proteins may lose their bioactivity during immobilization due to damage in the active sites and bioactive functional groups from the chemical binding method.

Mussel-inspired bioconjuctions. Observation of the natural phenomenon of the adhesion of mussels to a variety of material surfaces has led to an important advance in the area of biomedical science, specifically in the development of new drug delivery systems (Figure 1b2). Scientists have long noted that mussels can strongly attach to a diverse variety of substrates due to the formation of 3,4dihydroxy-L-phenylalanine (DOPA), which is the major active site of the extraordinarily robust adhesion near the plaque-material interface. ${ }^{33,34}$ On the basis of these investigations, Messersmith and his group found that dopamine can be easily deposited on virtually all types of organic and inorganic substrates to form a polydopamine layer with a molecular structure highly similar to that of DOPA. ${ }^{35}$ The primary advantages of polydopamine, as seen with mussels, are its robust and strong adhesion properties to virtually all types of surfaces in mild conditions, regardless of the surface's specific chemical properties, and the formation of reversible and noncovalent interlayers with high affinity and stability. ${ }^{36}$

Previous studies utilizing the reactivity of polydopamine films to immobilize GFs onto a large number of substrates provide a promising approach for the effective loading and sustained release of GFs, which ultimately improves tissue repair and implant integration. Polydopamine mainly tethered GFs to substrates through covalent bonds between the amino groups of GFs and the quinone groups (the catechol groups in dopamine can be oxidized into quinone groups) of polydopamine via Michael-type addition reactions and Schiff-base reactions. For example, Cho et al. ${ }^{37}$ developed polydopamine decorated poly(l-lactide) nanofibers for BMP-2 delivery, and surface chemical analysis confirmed that the successful tethering of BMP-2 was mediated by polydopamine films and that $\sim 90 \%$ of the BMP-2 was stably attached to the polymer surfaces for $>28$ days. The results suggest that this polydopamine modification method can be a useful tool for grafting biomolecules onto substrates, leading to improved bioactivity and accelerated tissue regeneration.
Other chemical coupling. Several other types of reactive groups with different chemistries have also been used to covalently attach GFs to biocompatible substrates. For instance, the surfaces of titanium alloy substrates were first functionalized with oxidized dextran, and BMP-2 was covalently linked to dextran-modified surfaces through a chemical conjugation between the amino-acid groups of proteins and the aldehyde groups of oxidized polysaccharides. ${ }^{38}$ Similarly, Leipzig et al. ${ }^{39}$ immobilized PDGF-AA or BMP-2 to a photopolymerizable methacrylic chitosan polymer through a streptavidin linker to guide neural stem cell differentiation in vivo. Another example demonstrated the use of parylene coatings in the sustainable immobilization of GFs via a maleimide-thiol coupling reaction under mild conditions. ${ }^{40}$ The site-specific approach defined in this study produced preferential bonds without damaging the tertiary structure of the GFs or compromising their biological activities.

The use of flexible, thin polymer brushes to covalently immobilize GFs has received considerable attention because their controlled polymer densities and nanometer size can facilitate better biological responses (Figure 1b3). Psarra et al. ${ }^{41}$ recently synthesized a poly (acrylic acid) polymer brush with a high grafting density to bind hepatocyte growth factor (HGF) and bFGF with a physisorption or chemisorption approach. Both GFs showed high loading efficiencies onto poly(acrylic acid) brushes based on their initial concentrations. The results indicated that the BMP-2-conjugated polymer brush could enhance adhesion and differentiation in terms of the alkaline phosphatase activity of MC3T3 cells on Ti surfaces. Therefore, polymer brushes biofunctionalized with GFs can be a promising method for creating enhanced cellular activity. In summary, while a variety of covalent conjugation approaches have been developed, a potential disadvantage of these methods is that GFs may lose a part or most of their bioactivity after covalent coupling.

\section{ECM-inspired GF delivery systems}

Heparin-based binding approach. Taking inspiration from the natural interactions between ECM and GFs, bioaffinity tethering approaches (Figure 1c1) have been designed for optimal delivery systems in a highly spatio-temporal controlled manner, further mimicking ECM functions. The ECM is a highly dynamic microenvironment that regulates multiple cellular processes; in addition, it acts as a reservoir for GFs due to their ability to bind multiple molecules with high affinity. In particular, many growth factors, such as BMP-2, BMP-7, VEGF, PDG and FGF-2, interact specifically with the heparin sulfate of the ECM. ${ }^{5,42}$ Therefore, a number of biomaterials have been decorated with heparin or heparin sulfate-mimetic molecules, sequestering the heparin-binding ability of GFs to improve their delivery. For example, Jha et al. $^{43}$ developed a series of heparin-functionalized hyaluronic acid-based hydrogels and investigated the effect of heparin molecular weight (MW) and its relative concentration on the loading efficiency and retention behavior of TGF $\beta 1$. The results demonstrated that high MW heparin facilitated TGF $\beta 1$ loading and retention and exhibited the slowest release kinetics, which was primarily due to its higher affinity for TGF $\beta 1$ compared to low MW heparin. More importantly, GF binding to high MW heparin decorated hydrogels and induced more robust differentiation of stem cells into endothelial cells, which further stimulated vascular-like network formation within the hydrogels.

Adhesive protein-based binding. On the other hand, several GF-binding motifs are present within ECM adhesive proteins, such as collagen, fibronectin, fibrinogen and vitronectin (Figure 1c2). ${ }^{44-46}$ In an ectopic bone defect model in the rat, hyaluronic acid hydrogel 
functionalized with the fibronectin fragment was designed for the delivery of BMP-2, and it promoted the formation of twice as much ectopic bone with a better production of collagen fibers compared to BMP-2 delivered via non-functionalized hyaluronic acid hydrogel. ${ }^{47}$ In a critical-sized calvarial defect model in the rat, dual-delivery of BMP-2 and PDGF-BB with the adhesive fibronectin fragment was able to accelerate bone formation at low doses, whereas the growth factors delivered without the adhesive proteins produced no significant effect. ${ }^{48}$ The two aspects of retained engineered GFs, along with natural biological adhesive proteins, may produce synergistic effects in nervous system reconstruction and repair.

ECM components and hierarchical structure-based binding. In addition to the bioaffinity of ECM components, the architecture of ECM microenvironments also plays a pivotal role in directly modulating GF activity and cell fate during tissue repair (Figure 1c3). Eap et al. ${ }^{49}$ constructed biomimetic ECM nanostructures for the immobilization of BMP-2 by assembling polysaccharides (chitosan) on collagen nanofibers. Both in vitro and in vivo evaluations confirmed that this sophisticated strategy enhanced the safety and efficacy of the therapeutic matrix in terms of bone formation compared to the more simplistic, traditional approaches based on immersion of the substrates with GFs. To comprehensively simulate ECM functions, our group has developed bioadhesive microporous architectures that mimic the functions of ECM through self-assembling polydopamine microcapsules and chitosan, which not only enhance osteoblast functions but also but also facilitate BMP-2 immobilization and provide sustained release in local sites (Figure 2) ${ }^{50}$ More importantly, the bioadhesive microarchitecture and its immobilized BMP-2 with both cell affinitive and high GF loading capacity synergistically enhanced the activity and osteogenetic differentiation of stem cells. Once bound to the ECM, the bioactivity and release kinetics of immobilized GFs are dependent on their binding-affinity, underlying micro/nano structures, and the use of multiple immobilization approaches.

\section{NANOCARRIERS FOR GF ENCAPSULATION AND RELEASE FOR BIOMEDICAL APPLICATIONS}

Synthetic polymer NPs

Biocompatible NPs have received considerable attention for drug delivery due to their small dimensions, high surface area to volume ratio, high drug loading efficiency, and ability to quickly respond to environmental stimuli, such as temperature, $\mathrm{pH}$, magnetic field or ultrasound (Table 2; Figure 3a). Synthetic polymers include polylactide (PLA), polyglycolide (PGA) and PLGA copolymers, which are wellsuited for NP fabrication to control drug release because of their established safety profile, long history of clinical applications, and wellunderstood degradation pattern. Among the different synthetic polymers developed to fabricate polymeric NPs, PLGA is one of the most successfully used synthetic polymers due to the following desirable properties: (1) FDA approval for clinical use in humans as a drug delivery system, (2) well-described formulations and methods of production adapted to various types of drugs, ranging from small molecules to macromolecules and (3) ability to protect drugs from degradation and the possibility of sustained release. ${ }^{51}$ Various methods include emulsion-solvent evaporation (single and double emulsion), phase separation, emulsification-solvent diffusion, solvent displacement, dialysis, spray drying and self-assembly, which have been extensively applied in the successful synthesis of PLGA NPs. ${ }^{52,53}$ The most common method used for the preparation of PLGA NPs is the emulsification-solvent evaporation technique. However, this method is primarily used for the encapsulation of hydrophobic drugs. A modification of this procedure called the double or multiple emulsion technique has become the favored protocol for encapsulating hydrophilic compounds and proteins. ${ }^{54}$

In addition, GFs directly adsorbed on hydrogels or scaffolds tend to exhibit rapid release in the short-term due to the open pore architecture and exposure of GFs to the bio environment, which weaken the bioactivity of GFs. As an indirect approach, GF-loaded NPs provide an effective approach to controlled GF release from scaffolds (Table 3)..$^{55}$ Shoichet et al. ${ }^{56}$ developed a novel delivery system that encapsulated EGF in PLGA NPs, followed by EGF polymeric particles dispersed in a hyaluronan methylcellulose (HAMC) hydrogel. The PLGA NPs effectively controlled EGF release within the first week and led to tissue repair in a mouse stroke model. Moreover, it has been reported that a poly(ether)urethane/polydimethylsiloxane/fibrin-based scaffold containing PLGA NPs loaded with VEGF and bFGF enhanced granulation tissue formation and maturity as well as collagen deposition when compared to other groups. ${ }^{57}$ One problem concerning PLGA is its poor affinity under physiological conditions, resulting in inadequate protein retention. Employing natural polymers or chemical crosslinking methods can enhance drug-loading ratios. In this delivery system, bFGF and VEGF were first encapsulated in PLGA-based NPs by a modified solvent diffusion method using heparin and human serum albumin as stabilizing agents.

\section{Protein-based NPs for GF delivery}

Albumin NPs have received considerable attention as drug delivery vehicles because they have several advantages (Figure $3 \mathrm{~b}$ ). First, the albumin molecule contains multiple drug binding sites, which leads to the high binding capacity of various drugs for albumin NPs. Specifically, due to their high content of charged amino acids (for example, lysine, arginine, glutamate and aspartate), albumin-based NPs are capable of loading either positively or negatively charged molecules by electrostatic forces without the addition of other accessory compounds. ${ }^{58,59}$ Second, their surface properties are well tolerated by charged polymers, which broaden the application of albumin NPs in biomedical areas. Third, albumin-based NPs are biodegradable, easy to fabricate, and reproducible under mild conditions by desolvation (coacervation), ${ }^{60}$ emulsification, ${ }^{61}$ and selfassembly techniques. ${ }^{62}$ It should be noted that desolvation is the most widely used method for the preparation of biomolecule-encapsulated albumin NPs.

In the coacervation process, albumin-based NPs are prepared by a continuous dropwise addition of ethanol to an albumin solution under continuous stirring until the solution becomes slightly turbid. During the addition of ethanol into the aqueous solution, albumin is phase separated due to its diminished water-solubility ${ }^{60}$ The BSA concentration, $\mathrm{pH}$ value of the aqueous solution, rate of ethanol addition, and ethanol to water ratio (the final volume ratio of ethanol added to the starting aqueous BSA solution) play key roles in determining the particle size and polydispersity of the resulting NPs. ${ }^{63}$ Since morphologically formed albumin particles are not sufficiently stabilized after dispersion in water, conventional albumin NPs prepared by this method often employ glutaraldehyde (GA) to stabilize them in which the amino moieties in lysine residues and arginine moieties in the guanidino chains of albumin are solidified by a covalent reaction with the aldehyde-group of glutaraldehyde. ${ }^{64}$

However, glutaraldehyde reacts with the free amines on albumin and possibly with any amines on the loaded biomolecules, which could adversely affect drug bioactivity. The potential toxicity of 
a

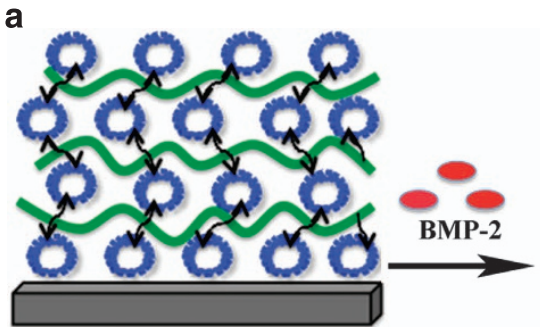

polydopamine capsule/chitosan

LbL films

e

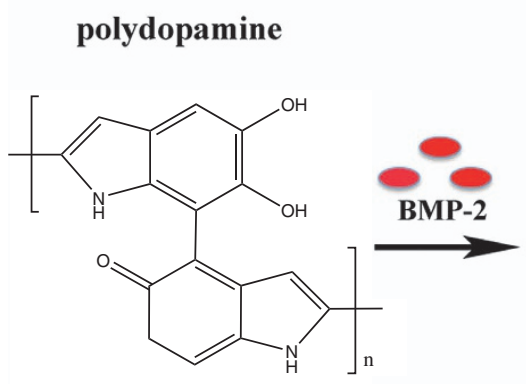

b

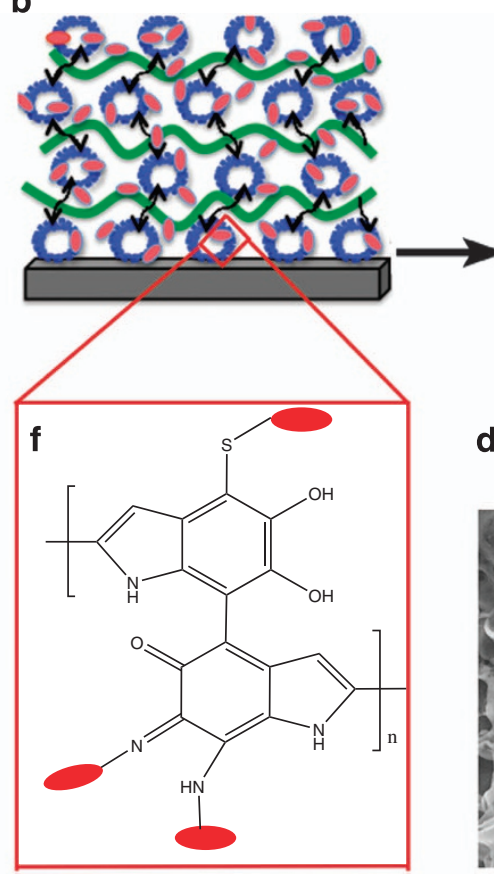

C

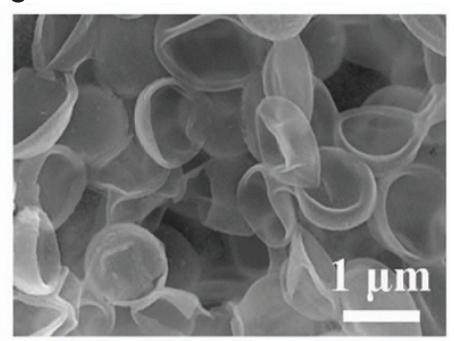

.

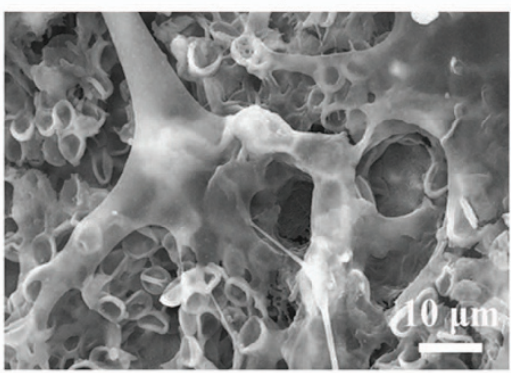

Figure 2 Schematic illustration of formation of polydopamine capsules/chitosan LbL films and subsequent loading and release of BMP-2 for enhanced cell adhesion. In detail, polydopamine capsules and chitosan self-assembly (a), BMP-2-loaded LbL films (b) Scanning electron microscopic (SEM) image shows polydopamine capsule-based architectures have highly interconnected micro-pore structures throughout the surfaces (c), BMSCs adhesion on LbL porous films (d), the structure of polydopamine molecules (e), the reaction mechanisms between polydopamine and BMP-2 in this system (free amino groups and thiol groups of BMP-2 and the catechol groups of polydopamine react with each other to form Schiff-base and Michael-type addition covalent bonds) (f).

glutaraldehyde is also a problem for in vivo delivery in this approach. As an alternative to glutaraldehyde, coating the protein NPs with cationic polymers provides a superior approach to stabilizing the particles and protecting the bioactivity of the encapsulated drug against enzymatic degradation. ${ }^{65}$ BMP-2 could be encapsulated in branched polyethylenimine (PEI) coated-BSA NPs by a conversion process, which resulted in an encapsulation efficiency that was typically $>90 \%$ in all NPs. ${ }^{66}$ In vitro release studies showed that the PEI concentration used in this delivery system efficiently controlled the release of BMP-2 in a gradual and sustained release pattern over a 10-day period. Furthermore, the higher PEI concentration realized a slower release of BMP-2 with a minimal burst release. It is hypothesized that the PEI coatings physically reduced BMP-2 diffusion through the NP surfaces; PEI stabilized the particles and protected the NP structure from disintegration. However, the osteoinductive activity and cytotoxicity of BMP-2 encapsulated in PEI coated-BSA NPs was a significant issue, and the PEI coatings would likely decrease BMP-2 activity. In addition, biocompatible polymers, such as PEIpolyethylene glycol (PEG) ${ }^{67}$ PEGylated multilayers ${ }^{68}$ and poly-L-lysine (PLL), ${ }^{65,69}$ are widely used to coat BSA NPs to effectively reduce their toxicity and enhance the bioactivity of their encapsulated drugs.

Polysaccharide-coated BSA NPs have been extensively employed as biomolecule reservoirs to construct biomimetic microenvironments for bone regeneration. Our previous publication demonstrated that chitosan and chitosan/oxidized alginate-coated BSA NPs were assembled on $\mathrm{Ti}$ surfaces to form biomimetic nanostructures (Figure 4). ${ }^{70}$ The nanostructured architectures exhibited a sustained release of BMP-2 and vancomycin over a long period, which was ascribed to the polysaccharide coatings that stabilize the BSA NPs in their bioenvironments. Bone marrow stromal cell (BMSC) culture tests indicated that the BSA NP-based architectures intrinsically facilitate the attachment, proliferation, and differentiation of BMSCs. Incorporating BMP-2 into the nanostructured architectures significantly promotes the osteogenetic differentiation of BMSCs, which reveals the synergistic effects of nanostructured architectures and BMP-2 on cell behaviors. Li et al. ${ }^{71}$ developed a new electrospun nanofiber scaffold that incorporated chitosan-coated BSA NPs for the controlled dual delivery of BMP-2 and dexamethasone (DEX). BMP-2-loaded BSA NPs were prepared in aqueous solution by the conversation technique and then stabilized by a chitosan layer though electrostatic interaction. Both the BMP-2-encopsulated BSA NPs and DEX were then incorporated into poly( $\varepsilon$-caprolactone)-poly(ethylene glycol) nanofibers through an electrospinning process. The dual-drugloaded nanofiber scaffold showed a sequential release model, which promoted calvarial defect repair in bone tissue engineering. Our research also confirmed that chitosan-decorated BMP-2-encapsulated (BSA) NPs immobilized on graphene oxide/chitosan scaffolds significantly enhanced bone marrow stromal cell functions and induced bone tissue regeneration. ${ }^{72}$

\section{Polysaccharide-based NPs}

Polysaccharides are comprised of multiple monosaccharide units joined together by glycosidic linkages (Figure 3c). ${ }^{73}$ Polysaccharides are the most attractive and promising natural polymers for the preparation of drug-encapsulated NPs due to their abundance in nature, low toxicity, high stability, low cost, biocompatibility, and presence of various functional groups on molecular chains. In particular, hydrophilic groups, such as amino, hydroxyl, or carboxyl groups, endow polysaccharides with multiple charges in aqueous solution. Among the widely investigated polysaccharides, chitosan is the most used cationic polymer, whereas alginate, heparin, chondroitin sulfate and hyaluronic acid are negatively charged. 
Charged polysaccharides can form physically crosslinked NPs in the presence of oppositely charged crosslinkers, which are either small molecules or polymers. Since ionically crosslinked NPs are readily prepared in mild conditions without the use of organic solvents at room temperature, they are promising agents for encapsulating more fragile drugs (for example, peptides and proteins) without significant loss of therapeutic function. Among polysaccharides, chitosan has been the most extensively investigated IC-NP as a carrier of small molecular drugs, proteins, and plasmid DNA, and it is prepared in the presence of the polyvalent anion tripolyphosphate (TPP). For example, Rajam et al. ${ }^{74}$ developed EGF- and FGF-incorporated chitosan/TPP NPs by the ionic gelation method for the sustained release of dual GFs. The drug loading capacity and loading efficiency of EGF or FGF in chitosan/TPP NPs were approximately $30 \pm 2.5 \mathrm{ng} \mathrm{mg}^{-1}$ of particles and $91 \pm 1.5 \%$, respectively. The cumulative release of EGF and FGF from the delivery systems was approximately $83 \%$ and $84 \%$, respectively, on day 35, without burst release in the initial stage, which greatly improved the attachment and proliferation of fibroblasts. Furthermore, the chitosan-based NP delivery system prepared by the ionic gelation method can also accommodate the multiple release model by chemical modifications. In another study by Butko et al..$^{75}$, bFGF was incorporated into chitosan/TPP and N-succinyl-chitosan/ TPP NPs by ionotropic gelation, and both types of NPs exhibited a similar entrapment efficiency of bFGF ( 60\%). The $N$-succinylchitosan/TPP NPs presented a larger and faster release of bFGF (18\%) for up to 7 days in comparison with chitosan/TPP NPs, which could be attributed to the higher swelling of $\mathrm{N}$-succinyl-chitosan/TPP NPs at $\mathrm{pH} 7.4$ in this study.

Recently, polysaccharide-based NPs prepared by a mild polyelectrolyte complex technique have received considerable attention for GF release due to their simple process and high affinity for GFs. Alonso and co-workers ${ }^{76}$ have prepared hyaluronic acid/chitosan NPs for the multifunctional delivery of two pro-angiogenic growth factors: VEGF and PDGF-BB. The resultant NPs had an average size of $200 \mathrm{~nm}$ and were able to efficiently encapsulate both growth factors, reaching association values of $94 \%$ and $54 \%$ for VEGF and PDGF, respectively. In vitro release studies indicated that PDGF-BB was released from the polysaccharide-based NPs in a sustained pattern over 1 week, whereas VEGF was completely released within 1 day.

However, chitosan-based NPs have also shown some disadvantages for GF release and tissue regeneration. In particular, chitosan has poor solubility in physiological conditions due to its strong intermolecular hydrogen bonding, thereby greatly restricting its biomedical application. Consequently, Tan et al. ${ }^{77}$ have prepared polysaccharide NPs utilizing the electrostatic interaction between $N, N, N$-trimethyl chitosan chloride (TMC) and low-molecular-weight heparin (LMWH) to achieve the sustained release of VEGF. In this delivery system, TMC has a higher solubility than that of chitosan in physiological conditions, which increases its applicability in tissue repair. In addition, LMWH can lead to less bleeding, and it exhibited a higher affinity for VEGF than that of normal heparin. These VEGF-loaded LMWHTMC NPs showed a spherical morphology with an average size of $146.5 \mathrm{~nm}$, and they were integrated in injectable nanofibrous hydrogel scaffolds in order to further decrease the burst release of VEGF at lower levels and increase the sustained release of VEGF in the longer term. The scaffolds significantly preserved the bioactivity of VEGF, thereby strongly promoting the proliferation and differentiation of adipose-derived stem cells for soft tissue engineering applications.

Heparin-based NPs have shown strong affinity and sustained release functions when coupled with various GFs, making them great candidates for emerging biomedical applications. ${ }^{78}$ For example, 


\section{a}
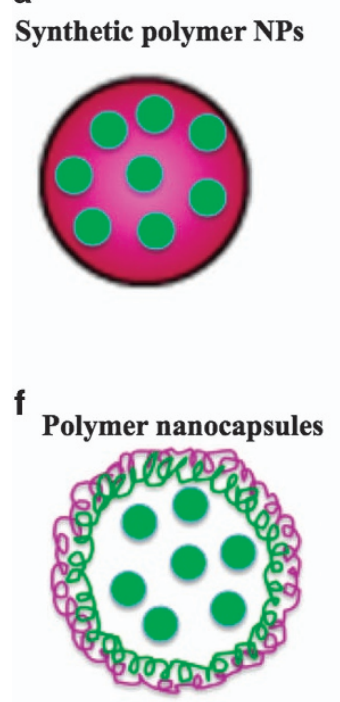

b

Protein-based NPs
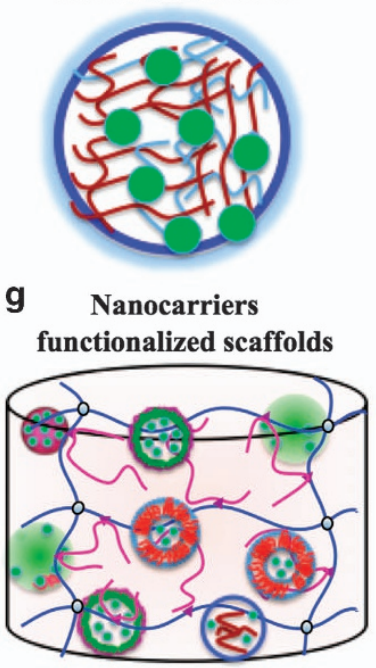

e

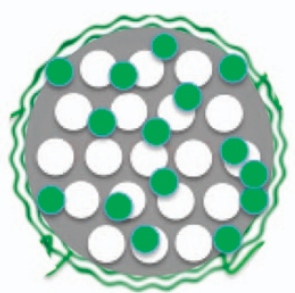

C

\section{Polysaccharide-based NPs}

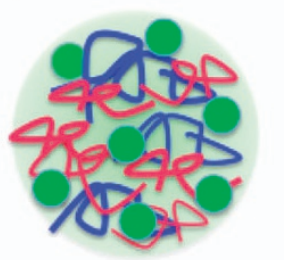

d Lipid-based NPs

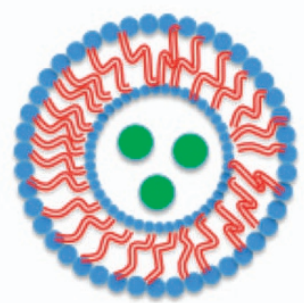

MSN-based NPs

Figure 3 Multiple nanocarriers for growth factors (GFs) encapsulation and release (a-f), GFs encapsulated nanocarriers functionalized biomaterials (g).

Tang et al. ${ }^{79}$ developed heparin-functionalized chitosan/poly $(\gamma$-glutamic acid) ( $\gamma$-PGA) NPs by a simple polyelectrolyte self-assembly method for the controlled release of bFGF. The NPs were formed through electrostatic interaction of the cationic chitosan and anionic $\gamma$-PGA and heparin, and the loading efficiency of bFGF increased significantly with the increase of encapsulated heparin in the particles. In another study, the role of heparin-based nanocomplexes encapsulating two GFs in the enhancement of osteoblast function were investigated. ${ }^{80}$ Approximately $80 \%$ of the PlGF- 2 and $90 \%$ of the BMP- 2 was stably retained in the nanocomplexes over a long period of time. In vitro biological assays indicated that the dual delivery of PlGF-2 and BMP-2 appears to have a synergistic effect on bone tissue regeneration in comparison to the delivery of either GF alone.

In addition, chondroitin sulfate is an anionic polysaccharide that is structurally similar to heparin, and it has a high binding affinity with positively charged GFs. In our previous study, ${ }^{81}$ BMP-2-encapsulated chitosan/chondroitin sulfate NPs were prepared through a simple and mild polyelectrolyte complexation method without involving harsh organic solvents or high temperature (Figure 5). NPs in the 482-698 nm mean size range were prepared, and the loading efficiency of BMP-2 in the particles was $\sim 91 \%$. These NPs were physically immobilized on biphasic calcium phosphate (BCP) scaffolds for the sustained release of growth factors. The protein-encapsulated NP on functionalized scaffolds exhibited a sustained release of protein over 40 days, whereas protein that was directly adsorbed onto the BCP scaffolds showed a burst release rate in the initial period. The longterm sustained release of protein could be ascribed to the slow degradation of the polysaccharide NPs and the stable binding force between the NPs and the surfaces of scaffolds. BMP-2 encapsulated NPs on BCP scaffolds induce more ectopic bone formation than bare
BCP scaffolds, which indicated the combination of a polysaccharidebased drug delivery system and porous scaffolds as a promising artificial bone substitute in clinical applications.

Lipid-based nanocarriers for controlled GF release

Lipid-based NPs were developed as advanced carriers for drug delivery due to their excellent amphiphilic properties, which originated from one or more phospholipid bilayers and facilitated their entrapment of lipophilic or hydrophilic drugs (Figure 3d) ${ }^{82,83}$ Lipid-based NPs are generally regarded as biocompatible and non-toxic because they are prepared with phospholipids derived from mammalian cell membranes. ${ }^{84}$ Three main configurations of lipid-based NPs are liposomes, solid lipid particles (SLN), and nanostructured lipid carriers (NLC). ${ }^{85}$ Liposomes are closed carriers formed by bilayers of hydrated phospholipids that enclose an aqueous core, which endows liposomes with amphiphilic properties and the ability to trap both hydrophobic and hydrophilic drugs. Liposomes can protect the activity of biomolecules against a range of environmental conditions, including extreme $\mathrm{pH}$, ionic strength and temperature. Therefore, several liposomes have been developed as EGF carriers for wound healing and bone repair. ${ }^{86,87}$ Furthermore, magnetic liposomes with incorporated BMP-2 or TGF- $\beta 1$ were prepared for enhanced bone or cartilage formation after topical injection. ${ }^{88,89}$ The NPs had an average size of $\sim 100 \mathrm{~nm}$ with an encapsulation efficiency of approximately $20 \%$ for BMP- 2 and TGF- $\beta 1$ in the liposomes. The results confirmed that both types of encapsulated GFs from the magnetic liposomes seemed to be cumulatively released at the target site over a prolonged period.

Nevertheless, liposomes have also shown several disadvantages, such as forming particle aggregates under aqueous conditions and spilling 
their contents in the short-term, as unsaturated lipids are susceptible to enzymatic dissociation. ${ }^{90}$ To address these issues, polymer coatings on liposomes provided an effective way to stabilize the particles and offered the desired release kinetics of encapsulated drugs. In addition, a three-dimensional LbL structure composed of xanthan and galactomannan biopolymers was assembled on dioctadecyldimethylammonium bromide liposome for EGF delivery, ${ }^{91}$ and it showed an entrapment efficiency of $72 \%$. The bilayer of biopolymer-coated liposomes $(165 \mathrm{~nm})$ increased the sustained delivery of EGF up to 5 times at a first order rate of $0.005 \mathrm{~min}^{-1}$. The use of these natural polysaccharide LbL coatings could be useful for improving biocompatibility, given the GF release profile of liposomes. In addition, EGFencapsulated multilamellar vesicle liposomes were fabricated to preserve the activity of biomolecules, and the encapsulation efficiency of EGF in the liposomes was $\sim 58 \% .^{92}$ The drug-loaded liposomes were further incorporated in chitosan gel to stabilize the structures of particles for enhanced wound healing. Overall, liposomes have a relatively low loading efficiency for GFs due to the incompatibility of lipids with proteins, which is the major obstacle in the widespread use of liposomes for GF delivery.

SLNs containing a solid lipid core were found to be an alternative carrier to liposomes due to their high stability in vivo. However, their low drug loading is an important drawback that could compromise future applications of this vehicle. Fortunately, recent investigations on lipid-based NPs (especially NLCs) have helped to improve SLNs and liposomes. NLCs with liquid lipid cores (solid lipids and oils) had an increased number of imperfections in the solid core matrix, thus facilitating the encapsulation of an increased amount of biomolecules while preserving the long-term stability of the nanocarriers. ${ }^{82,93}$ For instance, EGF-loaded SLN (332 nm) and NLC (348 nm) have been developed through the emulsification-ultrasonication technique for the treatment of chronic wounds. ${ }^{94}$ Characterization of the vehicles indicated that the encapsulation efficiency of EGF-loaded NLC was significantly higher than that of EGF-SLN. Moreover, the encapsulation process did not comprise the biological activity of EGF, as these lipid-based NPs improved the proliferation of keratinocytes and fibroblasts compared with free EGF. These data confirmed that NLC improved GF binding to the carriers and enabled sustained release of the GFs. Finally, these findings revealed that the EGF-lipid-based NPs, particularly EGF-NLC, could promote healing characteristics and maturation in terms of chronic wounds and re-epithelization grade. Recently, gelatin NLCs were prepared for the sustained release of bFGF $^{95}$ and NGF ${ }^{96}$ to enhance functional recovery in hemiparkinsonian rats and recovery in spinal cord injury, respectively. Altogether, the long-term stability, high loading efficiency, improved controlled release, and drug safety administration demonstrated by the use of NLCs makes them ideal candidates for drug delivery systems in clinical applications.

\section{Mesoporous silica NPs as GF carriers}

Mesoporous silica NPs (MSNs) or nanoporous silica NPs, have received considerable attention for biomedical applications because of their high specific surface area, controllable particle size, large pore volumes, accessible surface modifications and superior biocompatibility (Figure 3e). ${ }^{97-99}$ For example, BMP-2 peptides were grafted onto MSNs by covalently bonding through an aminosilane linker, and DEX was then incorporated into the channel of MSNs to construct dual delivery systems. ${ }^{100}$ The dispersibility and stability of MSNs in phosphate-buffered saline or cell medium were significantly improved by drug functionalization. In vitro results showed that the drug-loaded MSNs significantly promoted the osteogenic differentiation (ALP 
a

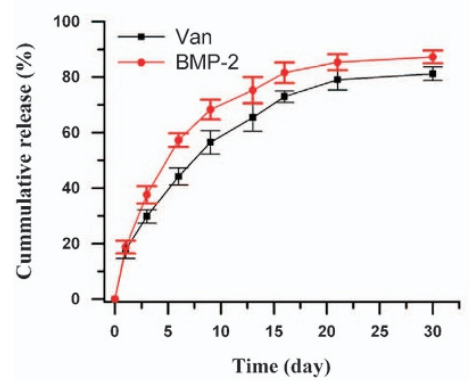

BMP-2 and Van release from polysacchride coated BSA-NPs b ${ }_{\mathrm{NH}_{3}}$

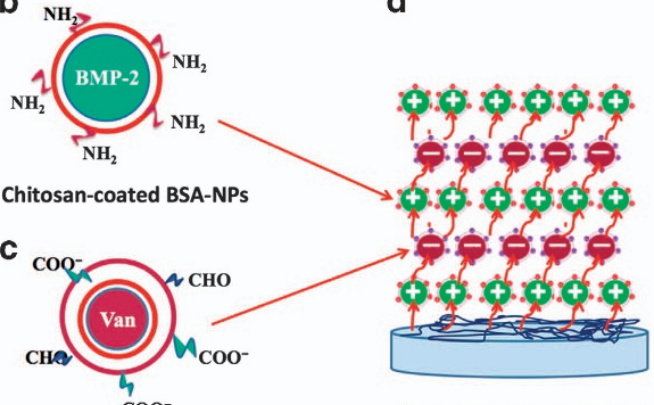

Porous nanostructures

Chitosan/oxidized alginate-coated BSA-NPs
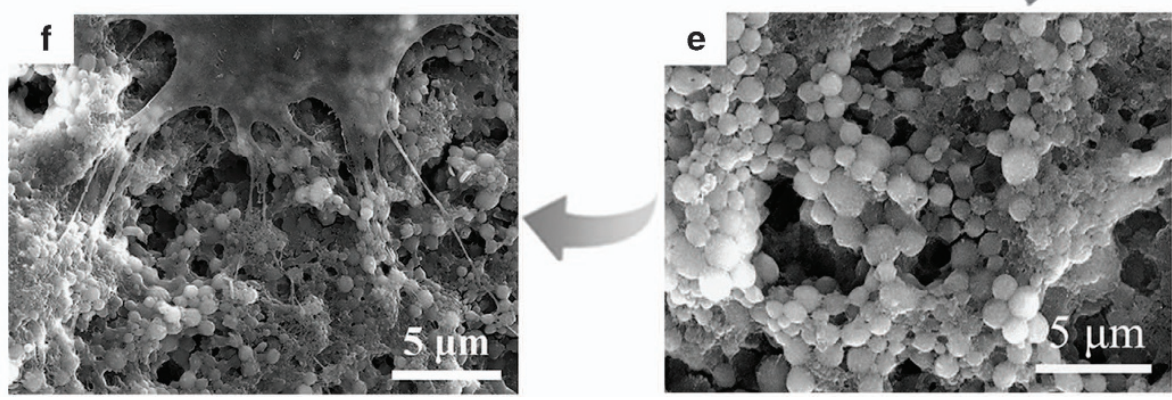

Figure 4 Schematic of bovine serum albumin (BSA)-based nanopaticle (NPs; BSA-NPs) assembled on titanium (Ti) surfaces for promoting cell adhesion. BMP-2 and vancomycin (Van) have sustained release up to 30 days from polysaccharide-coated BSA-NPs (a); Chitosan coated BMP-2 loaded BSA-NPs were prepared by a modified desolvation method (b); Chitosan/oxidized alginate coated Van loaded BSA-NPs were obtained by the same method (c); Cationic BSAbased NPs and anionic NPs were alternatively deposited on the Ti surfaces in a layer-by-layer technique (d); Surface morphologies of nanoporous structures by assembly of BSA-NPs on Ti surfaces (e); SEM image of BMSCs on nanostructured architectures (f). Bone marrow stromal cells (BMSCs) on nanostructured surfaces have close contact with the BSA-NPs, and present long thin pseudopodia similar to the size of NPs.

activity, calcium deposition) of BMSCs. Moreover, the MSNs-based dual delivery system synergistically induced effective osteoblast differentiation and ectopic bone formation in vivo, indicating that the MSNs have potential applications as bimolecular reservoirs in bone tissue engineering. It has also been reported that increasing the pore diameter of MSNs increases the drug delivery rate. ${ }^{101}$ Neumann et al. ${ }^{102}$ have prepared BMP2-functionalized nanoporous silicon NPs (NPSNPs) using a coupling method via an aminosilane linker. The NPs have an average size ranging from 30 to $50 \mathrm{~nm}$ (pore size $\sim 2.8 \mathrm{~nm}$ ), and the loading efficiency of BMP-2 loaded-NPSNPs was $\sim 0.99 \mathrm{\mu g} \mathrm{mg}^{-1}$. In vivo results confirmed that even BMP-free amino-modified NPSNPs show a certain osteoinductive effect, which, however, is significantly stronger with immobilized BMP-2 and can be further enhanced by supplementation with dexamethasone. Collectively, silica-based NPs are suitable as potential carriers for the controlled delivery of GFs in the area of bone tissue regeneration.

The surface decoration of MSNs with suitable polymers directly affects the drug release mechanism by enhancing drug diffusion resistance, which is an effective approach for achieving sustained drug release from MSNs. ${ }^{103}$ Previous studies have synthesized PEGdecorated MSNs with an encapsulated trypsin inhibitor, a model protein molecule for GFs. ${ }^{104}$ In vitro results indicated that protein release from the MSNs without the thin PEG film exhibited an initial burst followed by constant release, whereas the initial burst release of protein was completely inhibited in the case of the PEG-coated MSN system, and the release mechanism was found to have a zero-order release pattern over the entire period. Furthermore, recent developments in polymer coated-MSNs have enabled the programmable delivery of GFs by exploring stimulus-responsive polymers. Taken together, the surface modification of MSNs with functional polymers may enhance clinical drug outcomes by preserving their bioactivity and optimizing their release mode.

The combination of MSNs and porous scaffolds seems to be a promising platform for drug delivery in tissue engineering. Mehrasa et al. ${ }^{105}$ have fabricated PLGA/gelatin scaffolds containing MSNs by an electrospinning method; in vivo results demonstrated that the introduction of MNPs into the porous scaffolds improved the attachment, proliferation and formation of cellular processes of PC12 cells. Furthermore, the incorporation of MSNs into 3D porous scaffolds provided a potential way to encapsulate and deliver multiple drugs due to their biocompatibility and large-capacity storage of biomolecules. ${ }^{106,107}$ Lee et al. ${ }^{108}$ developed a new delivery system based on a collagen hydrogel containing NGF-loaded MSNs in neural tissue engineering. The MSN showed a high loading capacity and sustained release of NGF for over a week. When the NGF-loaded MSN was further incorporated in a collagen gel, the cumulative release amount of NGF was slower, and it followed the same long-term sustained release patterns. The novel delivery system of NGF-loaded MSNs, in combination with collagen hydrogels, promoted neuritogenesis while preserving cell viability, confirming that MSN embedded-porous scaffolds could also be extended to other GFs. Collectively, MSN-based delivery systems have been shown to be attractive candidates as GF carriers for tissue regeneration due to their excellent biocompatibility and large porosity. 


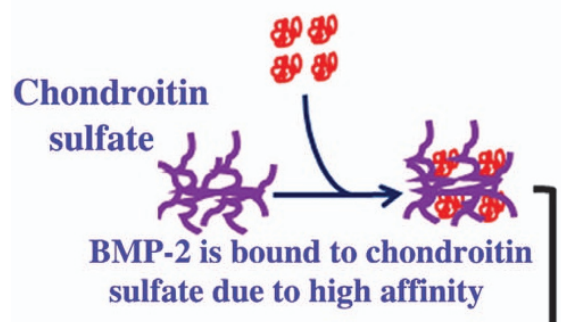

Chitosan/Chondroitin sulfate NPs

Polyelectrolyte complexation
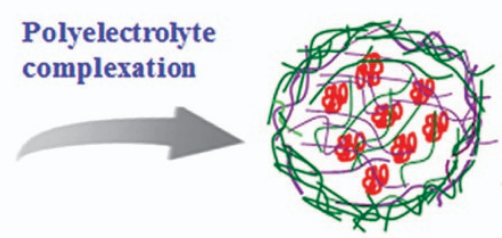

Chitosan
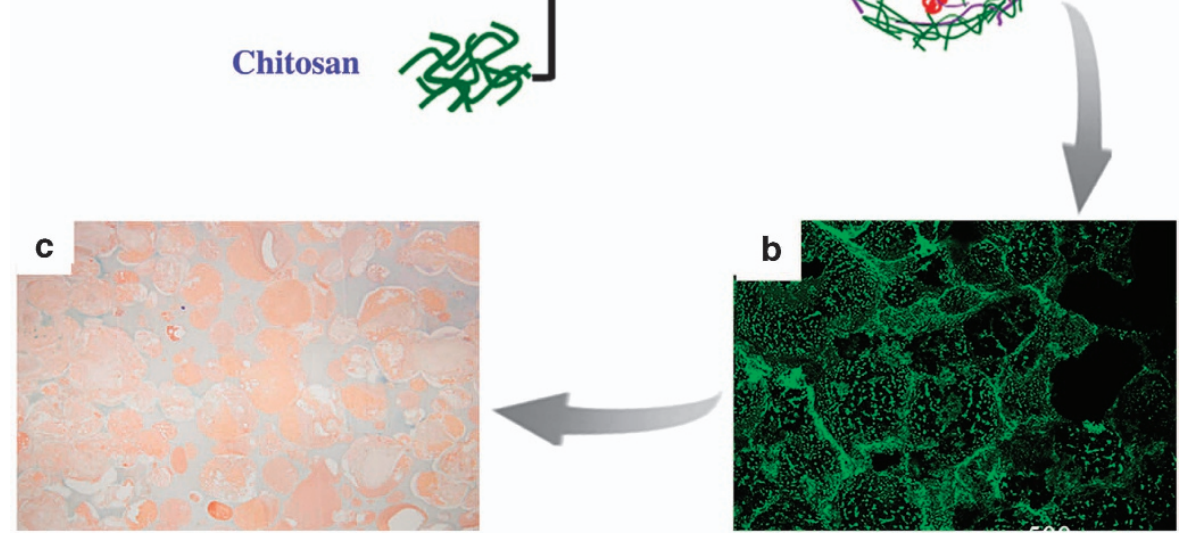

b

Figure 5 Schematic of the preparation process of BMP-2 encapsulated chitosan/chondroitin sulfate NPs and immobilization of the NPs on porous biphasic calcium phosphate (BCP) scaffolds for the study of ectopic bone formation. (a) Preparation of BMP-2 encapsulated chitosan/chondroitin sulfate NPs (a), FITC labeled chitosan/chondroitin sulfate NPs uniformly distributed on the BCP scaffold, as revealed by a confocal laser scanning microscope (CLSM) (b), Immunohistochemical staining for collagen type I on deparaffinized sections of BMP-2 encapsulated NPs functionalized BCP scaffolds: extensive immunoreactive area (brown) is observed (c).

\section{Polymer nanocapsules for controlled GF release}

Nanocapsule-based delivery systems have been extensively studied for biomedical applications because they offer a number of advantages, including low polymer content, high drug encapsulation efficiency, and polymeric shell protection against enzyme degradation (Figure 3f). ${ }^{109,110}$ Segura et al. ${ }^{111}$ have synthesized VEGF-loaded protein nanocapsules by assembling a thin polymer layer around the protein core by in situ free-radical polymerization. The introduction of peptides as crosslinkers to the polymer nanocapsules realized the celldemanded release rate of VEGF by mediating the catalytic hydrolysis of polymer shells via extracellular proteases. The sustained release of VEGF from nanocapsules significantly enhanced vascular formation in vitro and in vivo. Recently, BMP-2 loaded degradable protein nanocapsules were developed via in situ polymerization to increase effective osteogenisity and reduce side effects. ${ }^{112}$ Compared to the direct use of native BMP-2, the sustained release of BMP-2 from nanocarriers successfully enhanced bone formation with better bone quality while reducing the side effects associated with inconsistent bone formation and inflammatory edema in traditional spinal cord fusion surgery. The BMP-2 nanocarriers provided a safe and more effective GF therapy for bone tissue regeneration than native BMP-2. It has been reported that a combination of bioactive VEGF encapsulated the inner core of PLGA nanocapsules (diameter $<200 \mathrm{~nm}$ ) and superparamagnetic iron oxide NPs (SPIONs) embedded in PLGA nanoshells was synthesized as a therapeutic strategy to enhance angiogenesis. ${ }^{113}$ The PLGA nanocapsules showed high encapsulation efficiencies for both VEGF and SPIONs and exhibited sustained VEGF release over 14 days. In vitro studies confirmed that GF bioactivity in the polymer nanocapsules significantly increased the proliferation of human microvascular brain endothelial cells upon the release of VEGF. In addition, VEGF-loaded magnetic PLGA nanocapsules could be guided in pro-angiogenic treatments under an external magnetic field.

Biomimetic strategies capable of the sequential and spatiotemporal release of GFs and other signaling molecules in local sites may lead to improved cell functions and may accelerate effective tissue repair. For this purpose, biodegradable chitosan scaffolds containing various GFs loaded-nanocapsules with different degradation rates were prepared for enhanced bone tissue regeneration. ${ }^{114}$ In particular, faster degrading PLGA capsules (190-615 nm) and slower degrading poly (3-hydroxybutyrate-co-3-hydroxyvalerate) nanocapsules (255-712 nm) were separately loaded with BMP-4, PDGF and IGF-I and subsequently incorporated into chitosan scaffolds for the tunable release of multiple GFs in biometric microenvironments. The morphology of chitosan scaffolds with a sponge-like open porous microstructure had a pore size in the range of $100-200 \mu \mathrm{m}$. It was found that the dual, fast release of PDGF and BMP-4 achieved the highest proliferation rate of human mesenchymal stem cells and enhanced mineralization in vivo.

\section{Other nanocarriers for controlled GF release}

Recently, several NPs, such as inorganic, mussel-inspired or stimulusresponsive NPs, have received considerable attention for protein delivery due to their stable properties and versatile functions arising from their ease of handling and suitable surface chemistry. ${ }^{36,115-118}$ Shevtsov et al. ${ }^{119}$ developed EGF incorporated dextran-coated super paramagnetic iron oxide NPs (SPION) as a diagnostic agent for increasing magnetic resonance imaging contrast. The EGF functionalization increased the retention of NPs in tumor sites and exhibited 
high magnetic resonance contrast potential. However, these NPs were prone to accumulating at a specific site and their long-term toxicology should be further investigated. It should be noted that various inorganic nanoparticulate materials for drug delivery were associated with long-term toxicity, raising serious concerns. Among the many inorganic delivery systems, calcium phosphate $(\mathrm{CaP})$ NPs received special emphasis because of their superior biocompatibility and biodegradability and because $\mathrm{CaP}$ is the major constituent of bone and tooth. Ding et al. ${ }^{120}$ exploited hydroxyapatite (HA) NPs and silk fibroin to achieve effective loading and the programmable, sustained release of BMP-2. Their studies demonstrated that the combined system provided preferable microenvironments for the tunable release of BMP-2 and significantly enhanced bone regeneration. However, synthesis of GF-loaded CaP NPs and the maintenance of particle stability are critical challenges for biomedical applications. ${ }^{118}$

Temperature-sensitive drug delivery is among the most widely investigated stimulus-responsive nanocarriers, and these nanocarriers can be combined to form dual-drug delivery systems in programmable concentrations for specific times. Poly( $N$-isopropyl acrylamide) (PNIPAM) and its derivatives have become the most extensively studied synthetic polymers in biomedical applications due to their lower critical solution temperatures, which are near physiological conditions ${ }^{121}$ and are therefore suitable for mediating GF release in vivo. Kose et al. ${ }^{115}$ have synthesized PNIPAM and PLGA nanocarriers for the encapsulation of IGF-I and TGF- $\beta 1$, respectively.

Taking inspiration from adhesive mussel proteins that facilitate the adsorption of multiple biomolecules and cell behaviors, our group recently developed polydopamine NPs with a uniform size distribution $(\sim 260 \mathrm{~nm})$ and a high affinity toward a variety of soluble proteins and peptides to decorate various scaffolds, including bio-ceramic, metallic, and polymer-based scaffolds (Figure 6). ${ }^{122}$ Interestingly, the biodegradable polydopamine NPs enhance BMP-2 adsorption on porous TCP scaffolds, and they realized the sustained release of BMP-2 in vitro for up to 30 days on scaffolds. Furthermore, polydopamine with immobilized BMP-2 synergistically enhances the adhesion, proliferation and osteogenetic differentiation of BMSCs and induces ectopic bone formation in rats. In our previous study, we developed EGF-loaded polydopamine NPs/PNIPAM hydrogels to enhance cell affinity, adhesive strength, and tissue adhesiveness. ${ }^{123}$ The results confirmed that incorporating polydopamine in the hydrogel helps to graft and sustain the release of EGF, which was ascribed to the high affinity of polydopamine to proteins via covalent-based reactions. In summary, the GF-grafted polydopamine NPs combined with porous scaffolds exhibited high cell affinity and bioactivity in vivo, which might have potential applications for tissue regeneration.

\section{CONCLUSIONS AND PERSPECTIVES}

When delivering GFs to promote tissue regeneration, the most important issue is to obtain optimal concentrations and gradients of the suitable GFs for precise spatial/temporal demands. To date, significant progress has been made in understanding fundamental information about the dynamic microenvironment and the biological properties of GFs to further develop effective GF-based delivery systems for the spatiotemporal regulation of protein delivery. A wide variety of direct and indirect approaches have been employed in recent decades, such as covalent conjunctions, ECM-inspired binding, and NP-based delivery systems, to mimic ECM environments to facilitate tissue repair. Most of the delivery systems presented in this review demonstrated enhanced stability and therapeutic potential of the GFs upon immobilization when compared to traditional regenerative medicine and drug delivery systems. It should be noted that
NP-based GF delivery systems have demonstrated several advantages due to their high surface area, nanostructures, and precise control of multiple proteins that play pivotal roles in the regulation of cellular adhesion, proliferation, differentiation and extracellular matrix production. Specifically, the nanotoxicology and related toxicity issues such as cytotoxicity, ecotoxicity and genotoxicity are of crucial importance to enhance their potential applications in clinical trials. The details of toxicity evaluation of different GF nanocarriers are provided in Supplementary Information. Furthermore, these nanocarriers can be combined with microporous scaffolds via advanced nanotechnology techniques in order to mediate GF release kinetics in a predesigned manner to increase tissue healing and regeneration (Figure 3g).

A major challenge to the successful delivery of GFs in the clinic is that considerable amounts of biomolecules are required during tissue regeneration. Spatially controlled GF presentation will continue to play a critical role in tissue engineering strategies and will be coupled with biomaterials that permit tunable release profiles to achieve true spatiotemporal regulation during delivery. Importantly, for this approach to realize its full potential, continued efforts to understand native biological signaling pathways during tissue development and healing will be necessary. This requirement includes recapitulating the concentrations and spatial and temporal distributions of bioactive factors during these processes, accounting for the effects of cellular heterogeneity, the construction of native tissue at high resolution and their carriers on target cells. Such knowledge could serve as engineering design criteria to specifically guide the release of GF from the delivery system. Thus, the delivery system should not only release the appropriate GFs at accurate doses and kinetics; it should also offer insights on multiple GFs and their native microenvironments during tissue healing in vivo. Once these elements are taken into account, the expanding variety of GFs can hold great promise for future therapies in clinical regenerative medicine.

GF delivery from a tremendous number of biomaterials has provided satisfactory results in bone fractures and fibular defects, improvements in Alzheimer's disease and periprosthetic fractures, and advances in peripheral nerve regeneration, dermal healing, and skeletal muscle regeneration. Significant advances have optimized the delivery of GFs to target tissues and facilitated their progression through clinical trials to reach the market. In addition, an osteoconductive $\beta$-TCP matrix/PDGF-BB delivery system was developed to reach a long duration of PDGF-BB for dental therapy in clinical applications by utilizing innovative tissue engineering and drug encapsulation principles. However, supraphysiological and high doses of these GFs are required for therapeutic benefits, and in some cases, these doses have triggered certain side effects, including increased ectopic tissue formation, wound complications, radiculopathy, or cancer risk, from systemic exposure to the administered GFs. ${ }^{124}$ In addition, although sophisticated release profiles can now be achieved for research purposes and some commercially available GF products have been used in humans, clinical trials on release technology and the results of many such systems have been largely unsatisfactory or at least suboptimal. These limitations are primarily due to their simple design, poor drug release mechanism, biomaterial selection and matrix/carrier fabrication method, which make it is hard to mimic natural tissue regeneration.

Taking these points into account, it is clear that further research is necessary regarding the programmable release of growth factor delivery and smart biomaterial design. A combination of growth factors (BMP/VEGF, BMP-2/BMP-7) and their release profiles in different biomaterials has the potential to improve tissue regeneration 

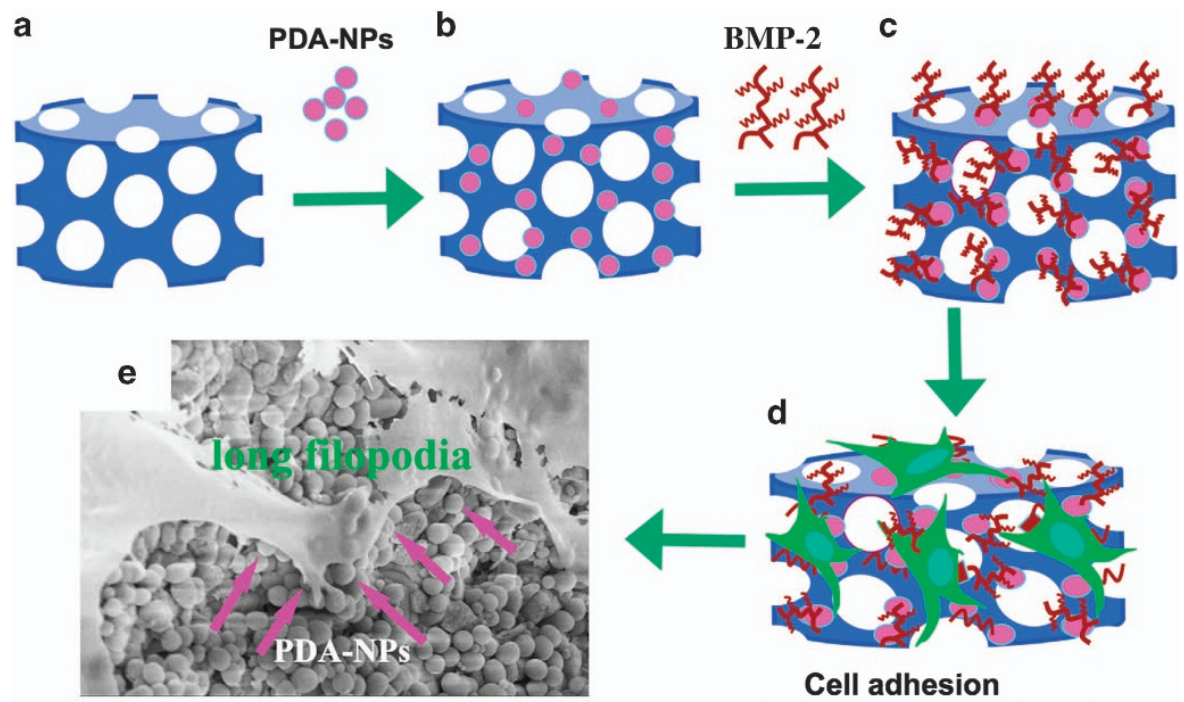

Cell adhesion
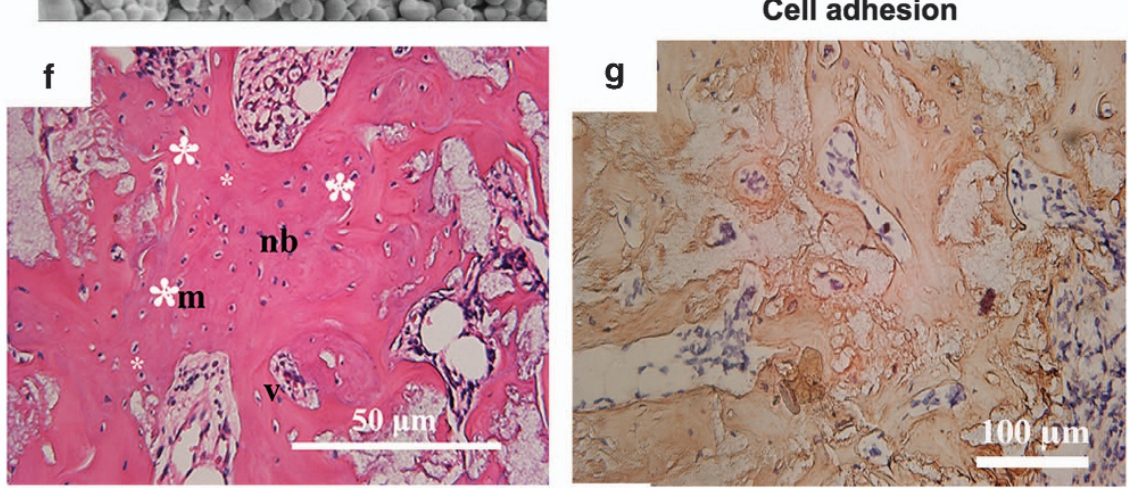

Figure 6 Polydopamine NPs functionalized on porous scaffolds for BMP-2 immobilization and enhanced bone tissue regeneration. (a) Porous TCP scaffolds; (b) polydopamine NPs immobilized on scaffolds; (c) BMP-2 adsorption on polydopamine NP-modified scaffolds (polydopamine-NP-TCP); (d) In vitro cell culture on porous scaffolds; (e) Attachment and spreading of BMSCs on polydopamine-NP-TCP scaffolds after 1 day; (f) Hematoxylin and eosin (H\&E) staining micrographs of polydopamine-NP-TCP scaffolds retrieved after 12-week implantation; (g) Immunohistochemical staining for collagen type I on deparaffinized sections of polydopamine-NP-TCP scaffolds retrieved after 12-week implantation. m, material; nb, new bone; v, vessel.

in vivo. Thus, multiple GF release plays an essential role in the recruitment, proliferation, and functional activities of MSCs during the early phases of wound repair and the promotion of tissue regeneration. Second, while a number of GFs have been demonstrated to be efficient at high doses in multiple biomedical applications, safety and precision medical treatment have driven research towards the development of novel delivery systems that enable dose reduction and optimize the concentrations of the right GFs at the right time. Therefore, smart nanomaterials that are sensitive to physiological or exogenous stimuli have been widely applied to deliver GFs at the right time and/or place. Indeed, the development of smart materials, mainly based on ECM-like biomaterials, appears to provide an attractive alternative for delivering multiple GFs in a better-controlled fashion in the future.

As the field of tissue regeneration moves towards the design of multifunctional platforms to better recapitulate the complex process of natural tissue repairing, combinations of bioactive proteins with appropriate biomaterial carriers will direct innovation. To date, biomaterial-based GF delivery systems derived from synthetic, natural, or inorganic materials in various structures can provide differential immobilization efficiency and release kinetics, which enables the most successful release of multiple GFs with biological activity in the local environment. These new methods of biomaterial synthesis are still in the initial stages of lab research and development, lacking consistent clinical data. Future research directions for this type of biomaterial in tissue repair and regeneration should be focused on a comprehensive understanding of biomaterial-tissue interactions and the optimization of their chemicophysical properties for long-term performance. Therefore, the elegant synthesis of biomaterials in terms of biological compatibility and structural components that mimic native tissue repair through the integration of multiple areas, including biochemical stimuli (such as GFs, adhesive proteins, genes or functional groups) and physical cues (micro/nano-architectures, stimuli-responsive prosperities, stiffness, shear stress, hydrophobic and hydrophilic properties), is important for the development of next-generation biomedical devices. Finally, exploration of the potential of GF-based delivery systems for clinical use strongly depends on more researchers and multidisciplinary approaches that combine engineering, biomaterials, medicine, and the technical expertise of medical specialists. Close cooperation among experts in materials science, tissue engineering, chemistry and medicine may eventually bridge the gap between current research and clinical applications, and it is likely that multiple GF delivery strategies will provide many therapeutic benefits in the treatment of complex wounds and pathologies in the near future.

\section{CONFLICT OF INTEREST}

The authors declare no conflict of interest. 


\section{ACKNOWLEDGEMENTS}

This work was supported by the Natural Science Foundation of China (81371989, 81702162), Guangdong Science and Technology Department Project (2015A030313776 and 2016A050503008), and Shenzhen Municipal Science and Technology Innovation Committee Project (JCYJ20160229172757249, JCYJ20160301151248779, JSGG20150331154931068, CXZZ20151015151249563, CXZZ20150401152251209, GJHZ20140416123146582 and JCYJ20140416122812013).

\section{PUBLISHER'S NOTE}

Springer Nature remains neutral with regard to jurisdictional claims in published maps and institutional affiliations.

1 Barrientos, S., Stojadinovic, O., Golinko, M. S., Brem, H. \& Tomic-Canic, M. Growth factors and cytokines in wound healing. Wound Repair Regen. 16, 585-601 (2008).

2 Tayalia, P. \& Mooney, D. J. Controlled growth factor delivery for tissue engineering. Adv. Mater. 21, 3269-3285 (2009).

3 Yancopoulos, G. D., Davis, S., Gale, N. W., Rudge, J. S., Wiegand, S. J. \& Holash, J. Vascular-specific growth factors and blood vessel formation. Nature 407 242-248 (2000).

4 Brandi, M. \& Collin-Osdoby, P. Vascular biology and the skeleton. J. Bone Miner. Res. 21, 183-192 (2006)

5 Martino, M. M. \& Briquez, P. S. Extracellular matrix-inspired growth factor delivery systems for bone regeneration. Adv. Drug Delivery Rev. 94, 41-52 (2015).

6 Fei, Y., Gronowicz, G. \& Hurley, M. M. Fibroblast growth factor-2, bone homeostasis and fracture repair. Curr. Pharm. Des. 19, 3354-3563 (2013).

7 Seeherman, H. \& Wozney, J. H. Delivery of bone morphogenetic proteins for orthopedic tissue regeneration. Cytokine Growth Factor Rev. 16, 329-345 (2005).

8 Fischer, J., Kolk, A., Pautke, C., Warnker, P. H., Plank, C. \& Smeets, R. Future of local bone regeneration-protein versus gene therapy. J. Cranio Maxill. Surg. 39, 54-64 (2011).

9 Kirker-Head, C. A. Potential applications and delivery strategies for bone morphogenetic proteins. Adv. Drug Delivery Rev. 43, 65-92 (2000).

10 Ripamonti, U. Soluble osteogenic molecular signals and the induction of bone formation. Biomaterials 27, 807-822 (2006).

11 Hollinger, J. O., Hart, C. E., Hirsch, S. N., Lynch, S. \& Friedlaender, G. E. Recombinant human platelet-derived growth factor: biology and clinical applications. J. Bone Joint Surg. Am. 90, 48-54 (2008).

12 Lee, J. \& Blaber, M. Increased functional half-life of fibroblast growth factor-1 by recovering a vestigial disulfide bond. J. Prot. Proteomics 1, 47-53 (2013).

13 Edelman, E. R., Nugent, M. A. \& Karnovsky, M. J. Perivascular and intravenous administration of basic fibroblast growth factor: vascular and solid organ deposition. Proc. Natl Acad. Sci. USA 90, 1513-1517 (1993).

14 Lazarous, D. F., Shou, M., Scheinowitz, M., Hodge, E., Thirumurti, V., Stiber, J. A., Lobo, A. D. \& Hunsberger, S. Comparative effects of basic fibroblast growth factor and vascular endothelial growth factor on coronary collateral development and the arterial response to injury. Circulation 94, 1074-1082 (1996).

15 Carragee, E. J., Chu, G., Rohatgi, R., Hurwitz, E. L., Weiner, B. K., Yoon, S. T., Comer, G. \& Kopjar, B. Cancer risk after use of recombinant bone morphogenetic protein-2 for spinal arthrodesis. J. Bone Joint Surg. Am. 95, 1537-1545 (2013).

16 Simons, M. \& Ware, J. A. Therapeutic angiogenesis in cardiovascular disease. Nat Rev. Drug Discov. 2, 863-872 (2003).

17 Lee, S. H. \& Shin, H. Matrices and scaffolds for delivery of bioactive molecules in bone and cartilage tissue engineering. Adv. Drug Delivery Rev. 59, 339-359 (2007).

18 Shi, J., Votruba, A. R., Farokhzad, O. C. \& Langer, R. Nanotechnology in drug delivery and tissue engineering: from discovery to applications. Nano Lett. 10 3223-3230 (2010).

19 Pina, S., Oliveira, J. M. \& Reis, R. L. Natural-based nanocomposites for bone tissue engineering and regenerative medicine: a review. Adv. Mater. 27, 1143-1169 (2015).

20 Chen, F., An, Y. Zhang, R. \& Zhang, M. New insights into and novel applications of release technology for periodontal reconstructive therapies. J. Control. Release 149 , 92-110 (2011).

21 Murphy, W. L., Peters, M. C., Kohn, D. H. \& Mooney, D. J. Sustained release of vascular endothelial growth factor from mineralized poly(lactide-co-glycolide) scaffolds for tissue engineering. Biomaterials 21, 2521-2527 (2000).

22 Freeman, I. \& Cohen, S. The influence of the sequential delivery of angiogenic factors from affinity-binding alginate scaffolds on vascularization. Biomaterials 30 2122-2131 (2009).

23 Ziegler, J., Anger, D., Krummenaner, F., Breitig, D., Fickert, S. \& Guenther, K. P. Biological activity of recombinant human growth factors released from biocompatible bone implants. J. Biomed. Mater. Res. A. 86, 89-97 (2008).

24 Reyes, R., De la Riva, B., Delgado, A., Hernández, A., Sánchez, E. \& Évora, C. Effect of triple growth factor controlled delivery by a brushite-PLGA system on a bone defect. Injury 43, 334-342 (2012).
25 Wang, Y., Angelators, A. S. \& Caruso, F. Template synthesis of nanostructured materials via layer-by-layer assembly†. Chem. Mater. 20, 848-858 (2007).

26 Richardson, J. J., Bjornmalm, M. \& Caruso, F. Technology-driven layer-by-layer assembly of nanofilms. Science 348, 411-424 (2015).

27 Macdonald, M. L., Samuel, R. E., Shah, N. J., Padera, R. F., Beben, Y. M. \& Hammomd, P. T. Tissue integration of growth factor-eluting layer-by-layer polyelectrolyte multilayer coated implants. Biomaterials 32, 1446-1453 (2011).

28 Shah, N. J., Macdonald, M. L., Beben, Y. M. \& Padera, R. F. Tunable dual growth factor delivery from polyelectrolyte multilayer films. Biomaterials 32, 6183-6193 (2011).

29 Min, J., Braatz, R. D. \& Hammond, P. T. Tunable staged release of therapeutics from layer-by-layer coatings with clay interlayer barrier. Biomaterials 35, 2507-2517 (2014).

30 Masters, K. S. Covalent growth factor immobilization strategies for tissue repair and regeneration. Macromol. Biosci. 11, 1149-1163 (2011).

31 Liu, L., Xing, Y., Li, S., Yuan, B., Chen, J. \& Xia, N. Activity analysis of the carbodiimide-mediated amine coupling reaction on self-assembled monolayers by cyclic voltammetry. Electrochim. Acta 89, 616-622 (2013).

32 Chiu, L. L. Y., Weisel, R. D., Li, R. K. \& Radisic, M. Defining conditions for covalent immobilization of angiogenic growth factors onto scaffolds for tissue engineering. J. Tissue Eng. Regener. Med. 5, 69-84 (2011).

33 Waite, J. H. \& Qin, X. Polyphosphoprotein from the adhesive pads of Mytilus edulis. Biochemistry 40, 2887-2893 (2001).

34 Harrington, M. J., Masic, A., Holten-Andersen, N., Waite, J. H. \& Fratzl, P. Iron-clad fibers: a metal-based biological strategy for hard flexible coatings. Science 328, 216-220 (2010)

35 Lee, H., Dellatore, S. M., Miller, W. M. \& Messersmith, P. B. Mussel-inspired surface chemistry for multifunctional coatings. Science 318, 426-430 (2007).

36 Liu, Y., Ai, K. \& Lu, L. Polydopamine and its derivative materials: synthesis and promising applications in energy, environmental, and biomedical fields. Chem. Rev. 114, 5057-5115 (2014).

37 Cho, H. J., Madhurakka Perikamana, S. K., Lee, J. H., Lee, J., Lee, K., Shin, C. \& Shin, H. Effective immobilization of BMP-2 mediated by polydopamine coating on biodegradable nanofibers for enhanced in vivo bone formation. ACS Appl. Mater. Interf. 6, 11225-11235 (2014).

38 Shi, Z., Neoh, K. G., Kang, E. T., Poh, C. \& Wang, W. Titanium with surface-grafted dextran and immobilized bone morphogenetic protein-2 for inhibition of bacterial adhesion and enhancement of osteoblast functions. Tissue Eng. A 15, 417-426 (2008).

39 Li, H., Koenig, A. M., Sloan, P. \& Leipzig, N. D. In vivo assessment of guided neural stem cell differentiation in growth factor immobilized chitosan-based hydrogel scaffolds. Biomaterials 35, 9049-9057 (2014).

40 Chen, Y. C., Sun, T. P., Su, C. T., Wu, J. T., Lin, C. Y., Yu, J. \& Huang, C. W. Sustained immobilization of growth factor proteins based on functionalized parylenes. ACS Appl. Mater. Interf. 6, 21906-21910 (2014).

41 Psarra, E., Foster, E., König, U., You, J., Ueda, Y., Eichhorn, K. J. \& Müller, M. Growth factor-bearing polymer brushes-versatile bioactive substrates influencing cell response. Biomacromolecules 16, 3530-3542 (2015).

42 Capila, I. \& Linhardt, R. J. Heparin-protein interactions. Angew. Chem., Inter. Ed. 41 390-412 (2002)

43 Jha, A. K., Mathur, A., Svedlund, F. L., Ye, J., Yeghiazarians, Y. \& Healy, K. E. Molecular weight and concentration of heparin in hyaluronic acid-based matrices modulates growth factor retention kinetics and stem cell fate. J. Control. Release 209, 308-316 (2015)

44 Upton, Z., Cuttle, L., Noble, A., Kempf, M., Topping, G., Malda, J. \& Xie, Y. Vitronectin: growth factor complexes hold potential as a wound therapy approach. J. Invest. Dermatol. 128, 1535-1544 (2008).

45 Martino, M. M., Briquez, P. S., Ranga, A., Lutolf, M. P. \& Hubbell, J. A. Heparin-binding domain of fibrin (ogen) binds growth factors and promotes tissue repair when incorporated within a synthetic matrix. Proc. Natl Acad. Sci. USA 110, 4563-4568 (2013).

46 Geiger, M., Li, R. H. \& Friess, W. Collagen sponges for bone regeneration with rhBMP-2. Adv. Drug Deliv. Rev. 55, 1613-1629 (2003).

47 Kisiel, M., Martino, M. M., Ventura, M., Hubbell, J. A., Hiborn, J. \& Ossipov, D. A. Improving the osteogenic potential of BMP-2 with hyaluronic acid hydrogel modified with integrin-specific fibronectin fragment. Biomaterials 34, 704-712 (2013).

48 Martino, M. M., Tortelli, F., Mochizuki, M., Traub, S., Ben-David, D., Kuhn, G. A \& Müller, R. Engineering the growth factor microenvironment with fibronectin domains to promote wound and bone tissue healing. Sci. Transl. Med. 3, 89-100 (2011).

49 Eap, S., Ferrand, A., Schiavi, J., Keller, L., Kokten, T., Floretti, F. \& Mainard, D. Collagen implants equipped with 'fish scale'-like nanoreservoirs of growth factors for bone regeneration. Nanomed. 9, 1253-1261 (2014).

50 Wang, Z., Li, C., Xu, J., Wang, K., Lu, X., Zhang, H., Qu, S., Zhen, G. \& Ren, F. Bioadhesive microporous architectures by self-assembling polydopamine microcapsules for biomedical applications. Chem. Mater. 27, 848-856 (2015).

51 Danhier, F., Ansorena, E., Sliva, J. M., Coco, R., Le Breton, A. \& Préat, V. PLGA-based nanoparticles: an overview of biomedical applications. J. Controlled Release 161, 505-522 (2012).

52 Chiellini, F., Piras, A. M., Errico, C. \& Chiellini, E. Micro/nanostructured polymeric systems for biomedical and pharmaceutical applications. Nanomed. 3, 367-393 (2008). 
53 Sharma, S., Parmar, A., Kori, S. \& Sandhir, R. PLGA-based nanoparticles: a new paradigm in biomedical applications. TrAC. Trends Anal. Chem. 80, 30-40 (2016).

54 Mao, S., Xu, J., Cai, C., Germershaus, O., Schaper, A. \& Kissel, T. Effect of WOW process parameters on morphology and burst release of FITC-dextran loaded PLGA microspheres. Int. J. Pharm. 334, 137-148 (2007).

55 Chen, F. M., Zhang, M. \& Wu, Z. F. Toward delivery of multiple growth factors in tissue engineering. Biomaterials 31, 6279-6308 (2010).

56 Wang, Y., Cooke, M. J., Sachewsky, N., Morshead, C. M. \& Shoichet, M. S. Bioengineered sequential growth factor delivery stimulates brain tissue regeneration after stroke. J. Controlled Release 172, 1-11 (2013).

57 Losi, P., Briganti, E., Errico, C., Lisella, A., Sanguinetti, E., Chiellini, F. \& Soldani, G. Fibrin-based scaffold incorporating VEGF-and bFGF-loaded nanoparticles stimulates wound healing in diabetic mice. Acta Biomater. 9, 7814-7821 (2013).

58 Kratz, F. Albumin as a drug carrier: design of prodrugs, drug conjugates and nanoparticles. J. Controlled Release 132, 171-183 (2008).

59 Irache, J. M., Merodio, M., Arnedo, A., Camapanero, M. A., Mirshahi, M. \& Espuelas, S. Albumin nanoparticles for the intravitreal delivery of anticytomegaloviral drugs. Mini Rev. Med. Chem. 5, 293-305 (2005).

60 Langer, K., Balthasar, S., Vogel, V., Dinauer, N., Von Briesen, H. \& Schubert, D. Optimization of the preparation process for human serum albumin (HSA) nanoparticles. Int. J. Pharm. 257, 169-180 (2003).

61 Crisante, F., Francolini, I., Bellusci, M., Martinelli, A., D'llario, L. \& Piozzi, A. Antibiotic delivery polyurethanes containing albumin and polyallylamine nanoparticles. Eur. J. Pharm. Sci. 36, 555-564 (2009).

62 Dai, L., Li, C., Liu, K., Su, H., Chen, B., Zhang, G. \& He, J. Self-assembled serum albumin-poly (I-lactic acid) nanoparticles: a novel nanoparticle platform for drug delivery in cancer. RSC Adv. 5, 15612-15620 (2015).

63 Rohiwal, S. S., Satvekar, R. K., Tiwari, A. P., Raut, A. V., Kumbhar, S. G. \& Pawar, S. H. Investigating the influence of effective parameters on molecular characteristics of bovine serum albumin nanoparticles. Appl. Surf. Sci. 334, 157-164 (2015).

64 Meziani, M. J. \& Sun, Y. P. Protein-conjugated nanoparticles from rapid expansion of supercritical fluid solution into aqueous solution. J. Am. Chem. Soc. 125, 8015-8018 (2003).

65 Wang, G., Siggers, K., Zhang, S., Jiang, H., Xu, Z., Zernicke, R. F. \& Matyas, J. Preparation of BMP-2 containing bovine serum albumin (BSA) nanoparticles stabilized by polymer coating. Pharm. Res. 25, 2896-2909 (2008).

66 Zhang, S., Wang, G., Lin, X., Chatzinikolaidou, M., Jennissen, H. P., Laub, M. \& Uludağ, H. Polyethylenimine-coated albumin nanoparticles for BMP-2 delivery. Biotechnol. Prog. 24, 945-956 (2008).

67 Zhang, S., Kucharski, C., Doschak, M. R., Sebald, W. \& Uludağ, H. PolyethyleniminePEG coated albumin nanoparticles for BMP-2 delivery. Biomaterials 31, 952-963 (2010).

68 Xie, L., Tong, W., Yu, D., Xu, J., Li, J. \& Gao, C. Bovine serum albumin nanoparticles modified with multilayers and aptamers for $\mathrm{pH}$-responsive and targeted anti-cancer drug delivery. J. Mater. Chem. 22, 6053-6060 (2012).

69 Singh, H. D., Wang, G., Uludağ, H. \& Unsworth, L. D. Poly-L-lysine-coated albumin nanoparticles: stability, mechanism for increasing in vitro enzymatic resilience, and siRNA release characteristics. Acta Biomater. 6, 4277-4284 (2010).

70 Wang, Z., Wang, K., Lu, X., Li, C., Han, L., Xie, C. \& Liu, Y. Nanostructured architectures by assembling polysaccharide-coated bsa nanoparticles for biomedical application. Adv. Health Mater. 4, 927-937 (2015).

71 Li, L., Zhou, G., Wang, Y., Yang, G., Ding, S. \& Zhou, S. Controlled dual delivery of BMP-2 and dexamethasone by nanoparticle-embedded electrospun nanofibers for the efficient repair of critical-sized rat calvarial defect. Biomaterials 37, 218-229 (2015).

72 Xie, C., Lu, X., Han, L., Xu, J., Wang, Z., Jiang, L. \& Wang, K. Biomimetic mineralized hierarchical graphene oxide/chitosan scaffolds with adsorbability for immobilization of nanoparticles for biomedical applications. ACS Appl. Mater. Interf. 8, 1707-1717 (2016).

73 Mizrahy, S. \& Peer, D. Polysaccharides as building blocks for nanotherapeutics. Chem. Soc. Rev. 41, 2623-2640 (2012).

74 Rajam, M., Pulavendran, S., Rose, C. \& Mandal, A. B. Chitosan nanoparticles as a dual growth factor delivery system for tissue engineering applications. Int. J. Pharm. 410, 145-152 (2011).

75 Butko, A., Bonat Celli, G., Paulson, A. \& Ghanem, A. Entrapment of basic fibroblast growth factor (bFGF) in a succinylated chitosan nanoparticle delivery system and release profile. J. Biomater. Sci. Polym. Ed. 27, 1045-1057 (2016).

76 Parajó, Y., d' Angelo, I., Welle, A., Garcia-Futentes, M. \& Alonso, M. J. Hyaluronic acid/Chitosan nanoparticles as delivery vehicles for VEGF and PDGF-BB. Drug Deliv. 17, 596-604 (2010).

77 Tan, H., Shen, Q., Jia, X., Yuan, Z. \& Xiong, D. Injectable nanohybrid scaffold for biopharmaceuticals delivery and soft tissue engineering. Macromo. Rapid Commun. 33, 2015-2022 (2012).

78 Tan, Q., Tang, H., Hu, J., Hu, Y., Zhou, X., Tan, Y. \& Wu, Z. Controlled release of chitosan/heparin NP-delivered VEGF enhances regeneration of decellularized tissueengineered scaffolds. Int. J. Nanomed. 6, 929-942 (2011).

79 Tang, D. W., Yu, S. H., Ho, Y. C., Mi, F. L., Kuo, P. L. \& Sung, H. W. Heparinized chitosan/poly( $\gamma$-glutamic acid) nanoparticles for multi-functional delivery of fibroblast growth factor and heparin. Biomaterials 31, 9320-9332 (2010).

80 Liu, Y., Deng, L., Sun, H., Xu, J., Li, Y., Xie, X. \& Zhang, L. Sustained dual release of placental growth factor-2 and bone morphogenic protein-2 from heparin-based nanocomplexes for direct osteogenesis. Int. J. Nanomed. 11, 1147-1158 (2016).
81 Wang, Z., Wang, K., Lu, X., Li, M., Liu, H., Xie, C., Meng, F., Jiang, O., Li, C. \& Zhi, W. BMP-2 encapsulated polysaccharide nanoparticle modified biphasic calcium phosphate scaffolds for bone tissue regeneration. J. Biomed. Mater. Res. A 103, 1520-1532 (2015).

82 Müller, R. H., Radtke, M. \& Wissing, S. A. Solid lipid nanoparticles (SLN) and nanostructured lipid carriers (NLC) in cosmetic and dermatological preparations. Adv. Drug Deliv. Rev. 54, 131-155 (2002).

83 Ramasamy, T., Kim, J. O., Yong, C. S., Umadevi, K., Rana, D., Jiménez, C., Campos, J., Ramalingam, M. \& Haidar, Z. S. Novel core-shell nanocapsules for the tunable delivery of bioactive rhEGF: Formulation, characterization and cytocompatibility studies. J. Biomater. Tissue Eng. 5, 730-743 (2015).

84 Zhang, S. \& Uludağ, H. Nanoparticulate systems for growth factor delivery. Pharm. Res. 26, 1561-1580 (2009).

85 Béduneau, A., Saulnier, P. \& Benoit, J.-P. Active targeting of brain tumors using nanocarriers. Biomaterials 28, 4947-4967 (2007).

86 Li, H., Song, J. H., Park, J. S. S. \& Han, K. Polyethylene glycol-coated liposomes for oral delivery of recombinant human epidermal growth factor. Int. J. Pharm. 258, 11-19 (2003).

87 Saddi, K.R.G.C., Alves, G. D., Paulino, T. P., Ciancaglini, P. \& Alves, J. B. Epidermal growth factor in liposomes may enhance osteoclast recruitment during tooth movement in rats. Angle Orthod. 78, 604-649 (2008).

88 Matsuo, T., Sugita, T., Kubo, T., Yasunaga, Y., Ochi, M. \& Murakami, T. Injectable magnetic liposomes as a novel carrier of recombinant human BMP-2 for bone formation in a rat bone-defect model. J. Biomed. Mater. Res. A 66, 747-754 (2003).

89 Tanaka, H., Sugita, T., Yasunaga, Y., Shimose, S., Deie, M., Kubo, T., Murakami, T. \& Ochi, M. Efficiency of magnetic liposomal transforming growth factor-beta 1 in the repair of articular cartilage defects in a rabbit model. J. Biomed. Mater. Res. A 73, 255-263 (2005).

90 Taylor, T. M., Weiss, J., Davidson, P. M. \& Bruce, B. D. Liposomal nanocapsules in food science and agriculture. Crit. Rev. Food Sci. Nutr. 45, 587-605 (2005).

91 Kaminski, G. A. T., Sierakowski, M. R., Pontaroto, R., dos Santos, L. A. \& de Freitas, R. A. Layer-by-layer polysaccharide-coated liposomes for sustained delivery of epidermal growth factor. Carbohydr. Polym. 140, 129-135 (2016).

92 Değim, Z., Çelebi, N. \& Alemdaroğlu, C. Evaluation of chitosan gel containing liposome-loaded epidermal growth factor on burn wound healing. Int. Wound. J. 8, 343-354 (2011).

93 Beloqui, A., Solinís, M. Á., Rodríguez-Gascón, A., Almeida, A. J. \& Préat, V. Nanostructured lipid carriers: Promising drug delivery systems for future clinics. Nanomed. Nanotechnol. 12, 143-161 (2016).

94 Gainza, G., Pastor, M., Aguirre, J. J., Villullas, S., Pedraz, J. L., Hernandez, R. M. \& Igartua, M. A novel strategy for the treatment of chronic wounds based on the topical administration of rhEGF-loaded lipid nanoparticles: In vitro bioactivity and in vivo effectiveness in healing-impaired $\mathrm{db} / \mathrm{db}$ mice. J. Controlled Release 185, 51-61 (2014).

95 Zhao, Y. Z., Li, X., Lu, C. T., Lin, M., Chen, L. J., Xiang, Q., Zhang, M., Jin, R. R., Jiang, X. \& Shen, X. T. Gelatin nanostructured lipid carriers-mediated intranasal delivery of basic fibroblast growth factor enhances functional recovery in hemiparkinsonian rats. Nanomed. Nanotechnol. 10, 755-764 (2014).

96 Zhu, S. P., Wang, Z. G., Zhao, Y. Z., Wu, J., Shi, H. X., Ye, L. B., Wu, F. Z., Cheng, Y., Zhang, H. Y. \& He, S. Gelatin nanostructured lipid carriers incorporating nerve growth factor inhibit endoplasmic reticulum stress-induced apoptosis and improve recovery in spinal cord injury. Mol. Neurobiol. 53, 4375-4386 (2016).

97 Feng, W., Zhou, X., He, C., Qiu, K., Nie, W., Chen, L., Wang, H., Mo, X. \& Zhang, Y. Polyelectrolyte multilayer functionalized mesoporous silica nanoparticles for $\mathrm{pH}$-responsive drug delivery: layer thickness-dependent release profiles and biocompatibility. J. Mater. Chem. B 1, 5886-5898 (2013).

98 Slowing, I. I., Vivero-Escoto, J. L., Wu, C.-W. \& Lin, V. S. Y. Mesoporous silica nanoparticles as controlled release drug delivery and gene transfection carriers. Adv. Drug Deliv. Rev. 60, 1278-1288 (2008).

99 Feng, W., Nie, W., He, C., Zhou, X., Chen, L., Qiu, K., Wang, W. \& Yin, Z. Effect of $\mathrm{pH}$-responsive alginate/chitosan multilayers coating on delivery efficiency, cellular uptake and biodistribution of mesoporous silica nanoparticles based nanocarriers. ACS Appl. Mater. Interf. 6, 8447-8460 (2014).

100 Zhou, X., Feng, W., Qiu, K., Chen, L., Wang, W., Nie, W., Mo, X. \& He, C. BMP-2 derived peptide and dexamethasone incorporated mesoporous silica nanoparticles for enhanced osteogenic differentiation of bone mesenchymal stem cells. ACS Appl. Mater. Interf. 7, 15777-15789 (2015).

101 Horcajada, P., Ramila, A., Perez-Pariente, J. \& Vallet-Regı, M. Influence of pore size of MCM-41 matrices on drug delivery rate. Micropor. Mesopor. Mater. 68, 105-109 (2004).

102 Neumann, A., Christel, A., Kasper, C. \& Behrens, P. BMP2-loaded nanoporous silica nanoparticles promote osteogenic differentiation of human mesenchymal stem cells. RSC Adv. 3, 24222-24230 (2013).

103 Wang, Y., Zhao, Q., Han, N., Bai, L., Li, J., Liu, J., Che, E., Hu, L., Zhang, Q. \& Jiang, T. Mesoporous silica nanoparticles in drug delivery and biomedical applications. Nanomed. Nanotechnol. 11, 313-327 (2015).

104 Bhattacharyya, S., Wang, H. \& Ducheyne, P. Polymer-coated mesoporous silica nanoparticles for the controlled release of macromolecules. Acta Biomater. 8, 3429-3435 (2012)

105 Mehrasa, M., Asadollahi, M. A., Ghaedi, K., Salehi, H. \& Arpanaei, A. Electrospun aligned PLGA and PLGA/gelatin nanofibers embedded with silica nanoparticles for tissue engineering. Int. J. Biol. Macromol. 79, 687-695 (2015). 
106 Qiu, K., Chen, B., Nie, W., Zhou, X., Feng, W., Wang, W., Chen, L., Mo, X., Wei, Y. \& He, C. Electrophoretic deposition of dexamethasone-loaded mesoporous silica nanoparticles onto poly(I-lactic acid)/poly( $\varepsilon$-caprolactone) composite scaffold for bone tissue engineering. ACS Appl. Mater. Interf. 8, 4137-4448 (2016).

107 Song, B., Wu, C. \& Chang, J. Dual drug release from electrospun poly(lactic-co-glycolic acid)/mesoporous silica nanoparticles composite mats with distinct release profiles. Acta Biomater. 8, 1901-1907 (2012).

108 Lee, J. H., Park, J. H., Eltohamy, M., Perez, R., Lee, E. J. \& Kim, H. W. Collagen gel combined with mesoporous nanoparticles loading nerve growth factor as a feasible therapeutic three-dimensional depot for neural tissue engineering. RSC Adv. 3 24202-24214 (2013).

109 Reis, C. P., Neufeld, R. J., Ribeiro, A. J. \& Veiga, F. Nanoencapsulation I. Methods for preparation of drug-loaded polymeric nanoparticles. Nanomed. Nanotechnology 2 8-21 (2006).

110 Anton, N., Benoit, J.-P. \& Saulnier, P. Design and production of nanoparticles formulated from nano-emulsion templates-a review. J. Controlled Release 128, 185-199 (2008).

111 Zhu, S. \& Segura, T. Cell-demanded VEGF release via nanocapsules elicits different receptor activation dynamics and enhanced angiogenesis. Ann. Biomed. Eng. 44, 1983-1992 (2016).

112 Tian, H., Du, J., Wen, J., Liu, Y., Montgomery, S. R., Scott, T. P. \& Aghdasi, B. Growth-factor nanocapsules that enable tunable controlled release for bone regeneration. ACS Nano 10, 7362-7369 (2016).

113 Carenza, E., Jordan, O., Martinez-San Segundo, P., Jiř́k, R., Starčuk, Z. Jr, Borchard, G., Rosell, A. \& Roig, A. Encapsulation of VEGF 165 into magnetic PLGA nanocapsules for potential local delivery and bioactivity in human brain endothelial cells. J. Mater. Chem. B 3, 2538-2544 (2015).

114 Saygun, I., Bal, V., Kızıltay, A., Yilgor Huri, P., Avcu, F., Üstün, K., Hasirci, V. \& Hasirci, N. Proliferation and differentiation of mesenchymal stem cells in chitosan scaffolds loaded with nanocapsules containing bone morphogenetic proteins-4, platelet-derived growth factor and insulin-like growth factor 1. J. Biomater. Tissue Eng. 4, 181-188 (2014).

115 Ertan, A. B., Yılgor, P., Bayyurt, B., Çalıkoğlu, A. C., Kaspar, Ç., Kök, F. N., Kose, G. T \& Hasirci, V. Effect of double growth factor release on cartilage tissue engineering. J. Tissue Eng. Regener. Med. 7, 149-160 (2013).

116 Mura, S., Nicolas, J. \& Couveur, P. Stimuli-responsive nanocarriers for drug delivery. Nat. Mater. 12, 991-1003 (2013).

117 Lu, Y., Sun, W. \& Gu, Z. Stimuli-responsive nanomaterials for therapeutic protein delivery. J. Controlled Release 194, 1-19 (2014).

118 Bose, S. \& Tarafder, S. Calcium phosphate ceramic systems in growth factor and drug delivery for bone tissue engineering: A review. Acta Biomater. 8, 1401-1421 (2012).

119 Shevtsov, M. A., Nikolaev, B. P., Yakovleva, L. Y., Marchenko, Y. Y., Dobrodumov, A. V., Mikhrina, A. \& Martynova, M. G. Superparamagnetic iron oxide nanoparticles conjugated with epidermal growth factor (SPION-EGF) for targeting brain tumors. Int. J. Nanomed. 9, 273 (2014).

120 Ding, Z., Fan, Z., Huang, X., Lu, Q., Xu, W. \& Kaplan, D. L. Silk-hydroxyapatite nanoscale scaffolds with programmable growth factor delivery for bone repair. ACS Appl. Mater. Interf. 8, 24463-24470 (2016).

121 Schild, H. G. Poly(N-isopropylacrylamide): experiment, theory and application. Prog. Polym. Sci. 17, 163-249 (1992).

122 Wang, Z., Wang, K., Zhang, Y., Jiang, Y., Lu, X., Fang, L., Gan, D., Lv, C., Zhang, H. \& Qu, S. Protein-affinitive polydopamine nanoparticles as an efficient surface modification strategy for versatile porous scaffolds enhancing tissue regeneration. Part. Part. Syst. Charact. 33, 89-100 (2015).

123 Han, L., Zhang, Y., Lu, X., Wang, K., Wang, Z. \& Zhang, H. Polydopamine nanoparticles modulating stimuli-responsive PNIPAM hydrogels with cell/tissue adhesiveness. ACS Appl. Mater. Interfaces 8, 29088-29100 (2016).

124 James, A. W., LaChaud, G., Shen, J., Astarian, G., Nguyen, V., Zhang, X., Ting, K. \& Soo., C. A review of the clinical side effects of bone morphogenetic protein-2. Tissue Eng., Part B 22, 284-297 (2016).

125 Kobayashi, T., Mizuta, M., Hiwatashi, N., Kishimoto, Y., Nakamura, T., Kanemaru, S. \& Hirano, S. Drug delivery system of basic fibroblast growth factor using gelatin hydrogel for restoration of acute vocal fold scar. Auris Nasus Larynx 44, 86-92 (2017)

126 Choi, J. W., Kim, Y. S., Park, J. K., Song, E. H., Park, J. H., Kim, M. S., Shin, Y. S. \& Kim, C. H. Controlled release of hepatocyte growth factor from MPE'G-b-(PCL-ran-PLLA) Diblock copolymer for improved vocal fold regeneration. Macromol. Biosci. 17, 1600163 (2017)

127 Uz, M., Sharma, A. D., Adhikari, P., Sakaguchi, D. S. \& Mallapragada, S. K. Development of multifunctional films for peripheral nerve regeneration. Acta Biomater. 56, 141-152 (2017).

128 Yadlapati, M., Biguetti, C., Cavalla, F., Nieves, F., Bessey, C., Bohluli, P., Garlet, G. P., Letra, A., Fakhouri, W. D. \& Silva, R. M. Characterization of a vascular endothelial growth factor-loaded bioresorbable delivery system for pulp regeneration. J. Endodont. 43, 77-83 (2017).

129 Crouzier, T., Ren, K., Nicolas, C., Roy, C. \& Picart, C. Layer-by-layer films as a biomimetic reservoir for rhBMP-2 delivery: controlled differentiation of myoblasts to osteoblasts. Small 5, 598-608 (2009).

130 Crouzier, T., Sailhan., F., Becquart, P., Guillot, R., Logeart-Avramoglou, D. \& Picart, C. The performance of BMP-2 loaded TCP/HAP porous ceramics with a polyelectrolyte multilayer film coating. Biomaterials 32, 7543-7754 (2011).

131 Peterson, A. M., Pilz-Allen, C., Kolesnikova., T. \& Mohwald, H. Growth factor release from polyelectrolyte-coated titanium for implant applications. ACS Appl. Mater. Interf. 6, 1866-1871 (2013).

132 Zhang, Z., Li, Q., Han, L. \& Zhong, Y. Layer-by-layer films assembled from natural polymers for sustained release of neurotrophin. Biomed. Mater. 10, 55-60 (2015).

133 Sharon, J. L. \& Puleo, D. A. Immobilization of glycoproteins, such as VEGF, on biodegradable substrates. Acta Biomater. 4, 1016-1023 (2008).

134 Grasman, J. M., Page, R. L. \& Pins, G. D. Design of an in vitro model of cell recruitment for skeletal muscle regeneration using hepatocyte growth factor-loaded fibrin microthreads. Tissue Eng. A 23, 773-783 (2008).

135 Zouani, O. F., Chollet, C. Guillotin, B \& Durrieu, M. C. Differentiation of preosteoblast cells on poly(ethylene terephthalate) grafted with RGD and/or BMPs mimetic peptides. Biomaterials 31, 8245-8253 (2010).

136 Lee, S. J., Lee, D. Y., Yoon, T. R., Kim, H. K., Jo, H. H., Park, J. S., Lee, J. H., Kim, W. D., Kwon, L. K. \& Park, S. A. Surface modification of 3D-printed porous scaffolds via mussel-inspired polydopamine and effective immobilization of rhBMP-2 to promote osteogenic differentiation for bone tissue engineering. Acta Biomater. 40, 182-191 (2016).

137 Chien, C. Y. \& Tsai, W. B. Poly(dopamine)-assisted immobilization of Arg-Gly-Asp peptides, hydroxyapatite, and bone morphogenic protein-2 on titanium to improve the osteogenesis of bone marrow stem cells. ACS Appl. Mater. Interf. 5, 6975-6983 (2013).

138 Banks, J. M., Mozdzen, L. C., Harley, B. A. C. \& Bailey, R. C. The combined effects of matrix stiffness and growth factor immobilization on the bioactivity and differentiation capabilities of adipose-derived stem cells. Biomaterials 35, 8951-8959 (2014)

139 Gnavi, S., Blasio, L., Tonda, T. C., Mancardi, A., Primo, L., Ciardelli, G., Gambarotta, G., Geuna, S. \& Perroteau, I. Gelatin-based hydrogel for vascular endothelial growth factor release in peripheral nerve tissue engineering. J. Tissue Eng. Regener. Med. 11, 459-470 (2017).

140 Kim, J., Lin, B., Kim, S., Choi, B., Evseenko, D. \& Lee, M. TGF- $\beta 1$ conjugated chitosan collagen hydrogels induce chondrogenic differentiation of human synoviumderived stem cells. J. Biol. Eng. 9, 1-11 (2015).

141 Mittermayr, R., Slezak, P., Haffner, N., Smolen, D., Hartinger, J., Hofmann, A., Schense, J., Spazierer, D., Gampfer, J. \& Goppelt, A. Controlled release of fibrin matrix-conjugated platelet derived growth factor improves ischemic tissue regeneration by functional angiogenesis. Acta Biomater. 29, 11-20 (2016).

142 Kim, S. E., Yun, Y. P., Shim, K. S., Park, K., Choi, S. W., Shin, D. H. \& Suh, D. H. Fabrication of a BMP-2-immobilized porous microsphere modified by heparin for bone tissue engineering. Colloids. Surf. B 134, 453-460 (2015).

143 Arisaka, Y., Kobayashi, J., Ohashi, K., Kim, K., Akiyama, Y., Yamato, M. \& Okano, T. A heparin-modified thermoresponsive surface with heparin-binding epidermal growth factor-like growth factor for maintaining hepatic functions in vitro and harvesting hepatocyte sheets. Regener. Ther. 3, 97-106 (2016).

144 Martino, M. M., Briquez, P. S., Guc, E., Tortelli, F., Kilarski, W. W., Metzger, S. Rice, J. J., Kuhn, G. A., Muller, R. \& Swartz, M. A. Growth factors engineered for super-affinity to the extracellular matrix enhance tissue healing. Science 343 , 885-888 (2014).

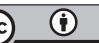

This work is licensed under a Creative Commons Attribution 4.0 International License. The images or other third party material in this article are included in the article's Creative Commons license, unless indicated otherwise in the credit line; if the material is not included under the Creative Commons license, users will need to obtain permission from the license holder to reproduce the material. To view a copy of this license, visit http:// creativecommons.org/licenses/by/4.0/

(C) The Author(s) 2017

Supplementary Information accompanies the paper on the NPG Asia Materials website (http://www.nature.com/am) 